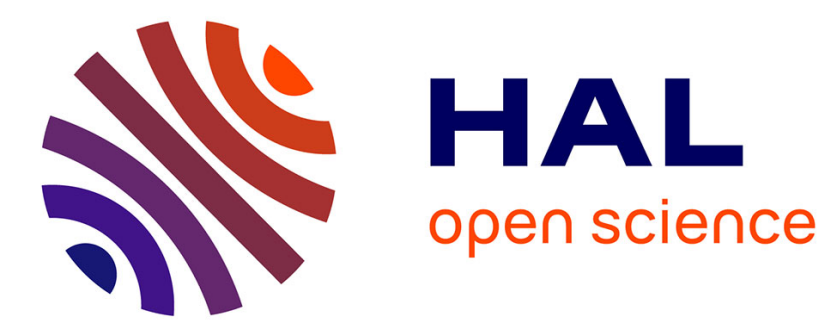

\title{
DÉTECTEURS A SEMICONDUCTEURS, PROGRÈS RÉCENTS
}

\author{
P. Siffert
}

\section{To cite this version:}

P. Siffert. DÉTECTEURS A SEMICONDUCTEURS, PROGRÈS RÉCENTS. Congress Of The French Physical Society, 1977, Poitiers, France. pp.C3-40-C3-56, 10.1051/jphyscol:1978308 . jpa00217412

\section{HAL Id: jpa-00217412 https://hal.science/jpa-00217412}

Submitted on 1 Jan 1978

HAL is a multi-disciplinary open access archive for the deposit and dissemination of scientific research documents, whether they are published or not. The documents may come from teaching and research institutions in France or abroad, or from public or private research centers.
L'archive ouverte pluridisciplinaire HAL, est destinée au dépôt et à la diffusion de documents scientifiques de niveau recherche, publiés ou non, émanant des établissements d'enseignement et de recherche français ou étrangers, des laboratoires publics ou privés. 


\title{
DÉTECTEURS A SEMICONDUCTEURS, PROGRÈs RÉCENTS
}

\author{
P. SIFFERT \\ Centre de Recherches Nucléaires, Groupe de Physique et Applications des Semiconducteurs (Phase), \\ 67037 Strasbourg Cedex, France
}

\begin{abstract}
Résumé. - On passe en revue les progrès récents ainsi que les problèmes qui se posent à l'emploi des détecteurs à semiconducteurs en spectrométrie $X$ et $\gamma$ ainsi qu'en détection d'ions lourds. Pour les premiers on se limite au tellurure de cadmium et à l'iodure mercurique, alors que pour les seconds, les compteurs minces $\Delta E / \Delta x$ à base de silicium sont plus spécialement étudiés. Finalement, les différents paramètres qui déterminent la réponse d'un compteur $E$ à base de silicium sont discutés.
\end{abstract}

\begin{abstract}
The recent evolution as well as the problems appearing in the use of semiconductor counters in both $\mathrm{X}$ and $\gamma$-ray as well as heavy ions spectroscopy are reviewed. For the photon counters the discussion is limited to cadmium telluride and mercuric iodide room temperature diodes, whereas for heavy ions, identification by means of thin $\Delta E / \Delta x$ counters and some problems related to the pulse amplitude in $E$ detectors are considered.
\end{abstract}

Introduction. - La spectrométrie nucléaire a beaucoup progressé avec l'avènement des détecteurs à semiconducteurs. Les diodes à base de germanium (pur ou compensé au lithium) sont largement employées à l'heure actuelle même en dehors de la physique nucléaire : par exemple en médecine nucléaire des appareils mettant en œuvre simultanément deux cents spectromètres Ge H.P. ont été développés [1]. Nous nous limiterons dans cet exposé aux détecteurs $\gamma$ ou $X$ réalisés à partir de matériaux nouveaux: le tellurure de cadmium (CdTe) et 1'iodure mercurique $\left(\mathrm{HgI}_{2}\right)$.

Par ailleurs, les possibilités et les limitations des diodes silicium (à barrière de surface ou compensées au lithium) en spectrométrie de particules chargées légères sont bien établies. Par contre, il nous a semblé que les problèmes nouveaux liés à la détection et à l'identification d'ions plus lourds ayant des énergies pouvant aller jusqu'à une centaine de MeV sont beaucoup moins connus. Nous nous intéresserons donc aux développements récents des compteurs $\Delta E / \Delta x$ minces et à quelques aspects de la spectroscopie d'ions lourds.

Spectrométrie $\gamma$ et $x$. - Le germanium et le silicium, qui constituent les matériaux actuellement employés pour la préparation de spectromètres de photons, avaient été retenus initialement à cause de leur important degré de développement technologique, consécutif aux besoins de l'industrie électronique. Ils ne constituent pas les semiconducteurs les mieux adaptés aux applications considérées ici. En effet, examinons brièvement les critères qui doivent être pris en considération pour le choix du matériau optimal :
- Numéro atomique Z. - Comme seules les interactions photoélectriques sont intéressantes et comme celles-ci augmentent rapidement avec le numéro atomique $Z$ du milieu absorbant, on cherchera évidemment à employer le matériau ayant le numéro atomique le plus élevé.

- Bande interdite Eg. - Pour permettre un fonctionnement à température ambiante avec un niveau de bruit (dû à la génération thermique de porteurs) minimal, il serait intéressant d'employer des cristaux ayant une valeur Eg élevée. Toutefois, lorsque cette énergie devient trop grande, la probabilité de piégeage et l'énergie requise pour créer une paire électron-trou deviennent prohibitives. Dans la pratique, les valeurs optimales se situent entre 1,5 et $2,2 \mathrm{eV}$.

- Densité de porteurs libres. - Pour un matériau de numéro atomique supérieur à 40 une épaisseur de $2 \mathrm{~mm}$ absorbera notablement les photons $\gamma$. En considérant qu'une telle zone de charge d'espace doit être obtenue pour une valeur raisonnable de la tension de polarisation (par exemple $500 \mathrm{~V}$ ), on peut calculer que le semiconducteur doit posséder moins de $10^{11}$ porteurs par $\mathrm{cm}^{3}$ à température ambiante (différence entre la concentration de trous et d'électrons).

- Vitesse de déplacement des porteurs. - Le temps de transit des porteurs à travers la zone sensible du compteur est inversement proportionnel à leurs mobilités. Dans les conditions de l'exemple précédent, une mobilité de $100 \mathrm{~cm}^{2} / \mathrm{V}$.s. conduit à des temps de transit de l'ordre de la microseconde. Plus il sera long, plus la probabilité de piégeage sera grande. 
- Densité de pièges, efficacité de collection. Les matériaux semiconducteurs et plus particulièrement les composés contiennent des centres de capture associés à la présence d'impuretés, de défauts de stoechiométrie ou de combinaisons de défauts et d'impuretés. Ces pièges, qui introduisent des niveaux dans la bande interdite, ont pour effet de retenir les porteurs pendant un temps plus ou moins long ce qui peut conduire à une perte de charges qui entraîne une dégradation de l'amplitude et de la résolution du compteur. Deux paramètres caractérisent ces phénomènes : la densité de pièges $N_{\mathrm{T}}$ et la durée de vie d'un porteur avant capture. On peut montrer que le piégeage n'a que peu d'influence si $N_{\mathrm{T}}<10^{10} \mathrm{~cm}^{-3}$ et la durée de vie $\tau>10^{-6} \mathrm{~s}$.

Quels sont les matériaux qui satisfont à l'ensemble de ces critères ?

Parmi le très grand nombre de cristaux semiconducteurs, il convient évidemment de se limiter à ceux qu'on est capable de préparer avec un degré de pureté et de perfection cristalline suffisant. Il ne subsiste alors que très peu de matériaux, qui sont reportés sur le tableau I. En fait, seul le tellurure de cadmium et l'iodure mercurique répondent aux critères énoncés ci-dessus. Nous allons les considérer plus en détail en négligeant volontairement les problèmes liés à la croissance et à la caractérisation de ces cristaux.

1. Tellurure de cadmium. - L'existence chimique du tellurure de cadmium est connue depuis le' siècle dernier [2] et dès 1911 un premier diagramme température-composition avait été établi [3]. Mais ce n'est qu'en 1954 que les propriétés semiconductrices ont été établies $[4,5]$. La contribution expérimentale la plus importante à ces premiers travaux est due à De Nobel [6]. Depuis cette date un grand nombre de recherches ont été entreprises tant pour établir les propriétés fondamentales de ce composé que pour évaluer ses applications possibles dans les domaines de l'optique (fenêtre I.R., modulateurs), de la conversion photovoltaïque (cellules solaires), de la détection nucléaire. Ces problèmes sont largement discutés dans les références $[7,8]$.

Pour atteindre des épaisseurs sensibles intéressantes il faut que les cristaux présentent des résistivités élevées, voire soient semi-isolants. Malheureusement, les deux procédés de croissance les plus employés, à savoir la fusion de zone et la croissance en solvant, conduisent à des cristaux ayant une concentration trop forte de défauts physiques, de sorte que leur résistivité est trop faible pour préparer des compteurs $\gamma$. Ces défauts, tels les lacunes de cadmium, les atomes de cadmium et de tellure interstitiels ainsi que des associations plus complexes pouvant prendre plusieurs états de charge, introduisent tout comme les impuretés chimiques dopantes, un ou plusieurs niveaux dans la bande interdite qui sont électriquement actifs. La concentration nette de centres ionisés ne représente qu'une balance entre accepteurs et donneurs. Pour la rendre minimale il conviendra de compenser rigoureusement les deux types de porteurs. En principe, trois méthodes permettent d'atteindre cet objectif :

12];

- le tirage en conditions stcechiométriques [9- le recuit après tirage $[6,13-17]$;

- l'introduction d'impuretés compensatrices [18-20], agissant de même manière que le lithium dans le germanium. Le chlore et l'indium sont les dopants les plus couramment employés. Les mécanismes de compensation par ce procédé, le plus couramment mis en œuvre, ont été étudiés en détail dans la littérature [6, 21-23].

Il apparaît donc que les propriétés du tellurure de cadmium sont sensibles à de nombreux paramètres. Dans la pratique, les résistivités les plus élevées sont atteintes après compensation par des donneurs.

1.1 Caractéristiques DE CES matériauX. 1.1.1 Résistivité. - Les différentes méthodes de croissance, les divers éléments utilisés pour la compensation, les conditions expérimentales

TABLEAU I

\begin{tabular}{|c|c|c|c|c|}
\hline $\begin{array}{c}\text { Semi- } \\
\text { conducteur }\end{array}$ & $\begin{array}{c}\text { Largeur de la } \\
\text { bande interdite } \\
(300 \mathrm{~K})(\mathrm{eV})\end{array}$ & $\begin{array}{l}\text { Mobilité des } \\
\text { électrons } \\
(300 \mathrm{~K}) \\
\left(\mathrm{cm}^{2}, \mathrm{~V}^{-1} \cdot \mathrm{s}^{-1}\right)\end{array}$ & $\begin{array}{c}\text { Mobilités des } \\
\text { trous } \\
(300 \mathrm{~K}) \\
\left(\mathrm{cm}^{2} \cdot \mathrm{V}^{-1} \cdot \mathrm{s}^{-1}\right)\end{array}$ & $\begin{array}{l}\text { Durée de vie des } \\
\text { électrons } \\
\text { (dans le type P) } \\
\text { (s) }\end{array}$ \\
\hline - & - & - & - & 一 \\
\hline Si & 1,12 & 1500 & 600 & $3 \times 10^{-3}$ \\
\hline $\mathrm{Ge}$ & 0,67 & 3900 & 1800 & $10^{-3}$ \\
\hline C (diamant) & 5,47 & 2000 & 1550 & $10^{-8}$ \\
\hline GaAs & 1,43 & 8500 & 420 & $10^{-7}$ \\
\hline GaP & 2,25 & 300 & 100 & $10^{-8}$ \\
\hline $\mathrm{CdS}$ & 2,42 & 300 & 50 . & $10^{-8}$ \\
\hline CdTe & 1,5 & $\sim 1000$ & $\sim 80$ & $10^{-6}$ \\
\hline InSb & 0,17 & 78000 & 750 & $10^{-7}$ \\
\hline $\mathrm{GaSb}$ & 0,67 & 4000 & 1400 & $10^{-8}$ \\
\hline InAs & 0,36 & $\sim 33000$ & 460 & \\
\hline InP & 1,27 & 4600 & 150 & \\
\hline AlSb & 1,52 & 200 & 550 & \\
\hline $\mathrm{HgI}_{2}$ & 2,1 & 100 & 4 & $10^{-7}$ \\
\hline
\end{tabular}

$\begin{array}{ccc}\begin{array}{c}\text { Durée de vie des } \\ \text { trous } \\ \text { (dans le type N) } \\ (\mathrm{s})\end{array} & \begin{array}{c}\text { Numéro } \\ \text { atomique }\end{array} & \begin{array}{c}\text { Energie de } \\ \text { création de } \\ \text { paire }(\mathrm{eV})\end{array} \\ \begin{array}{c}- \\ 3 \times 10^{-3}\end{array} & - & - \\ 10^{-3} & 32 & 3,61(300 \mathrm{~K}) \\ 10^{-8} & 6,96(90 \mathrm{~K}) \\ 10^{-7} & 31-33 & 13,2(300 \mathrm{~K}) \\ 10^{-8} & 31-15 & 4,27 \\ 10^{-8} & 48-16 & 7,8 \\ 10^{-6} & 48-52 & 4,43(300 \mathrm{~K}) \\ 10^{-7} & 49-51 & 1,2 \\ 10^{-8} & 31-51 & \\ & 49-33 & \\ & 49-15 & \\ 10^{-8} & 13-51 & \\ & 80-53 & 4,15(300 \mathrm{~K})\end{array}$


conduisent essentiellement à quatre types de cristaux (Fig. 1) :

- type $N$, résistivité $10^{6}-10^{9} \Omega . \mathrm{cm}$, tirage par épuisement de solution avec compensation à l'indium ;

- type $\mathrm{P}$, résistivité $10^{6}-10^{9} \Omega . \mathrm{cm}$, tirage en solvant THM avec compensation par le chlore ;

- type $P$, résistivité $10^{4}-10^{7} \Omega . \mathrm{cm}$, tirage en solvant THM, sans compensation chimique extérieure ;

- type $N$, résistivité 100-1000 $\Omega$. cm, tirage par fusion de zone, sans compensation chimique extérieure.

Remarquons dès à présent que ces valeurs de résistivités déterminées par les techniques classiques (van der Pauw, effet Hall) sont le plus souvent fort éloignées de celles permettant de calculer les
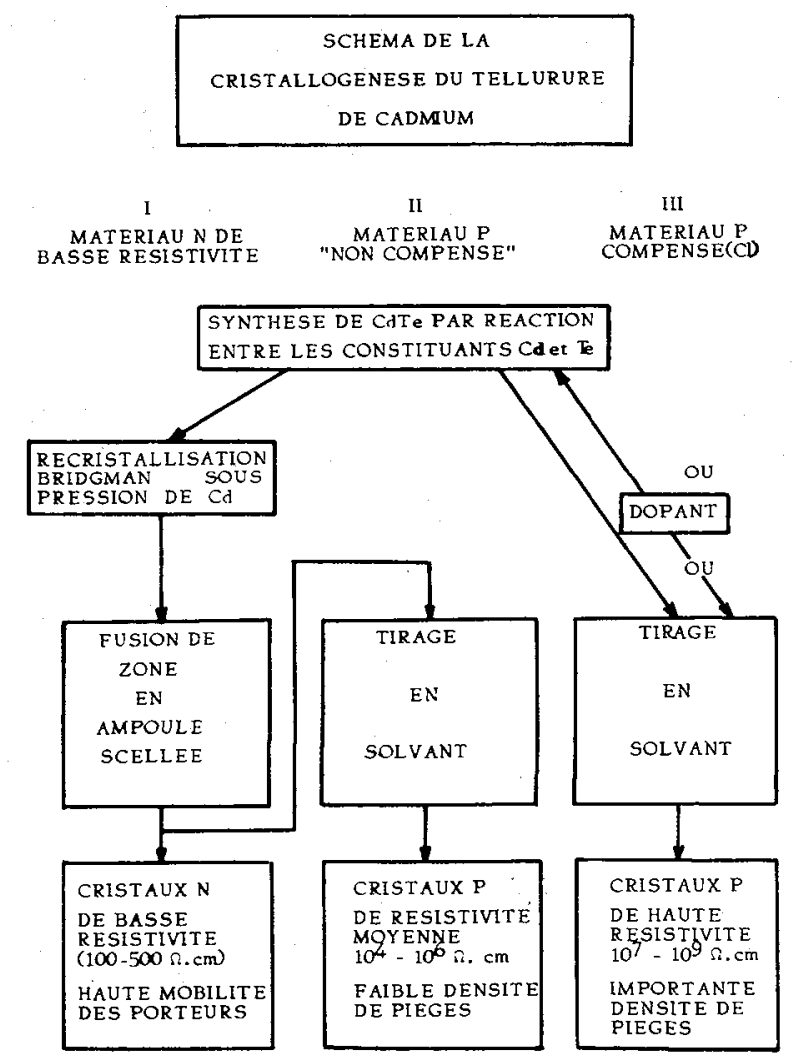

FIG. 1. - Schéma de la cristallogenèse des divers types de cristaux de tellurure de cadmium. épaisseurs de zone de charge d'espace. Ceci résulte d'une modification de la distribution des centres ionisés sous l'action du champ électrique appliqué au compteur.

1.1.2 Mobilité $\mu$ et vitesse des porteurs. - Plusieurs auteurs ont déterminé les paramètres de transport dans le tellurure de cadmium. En nous limitant aux mesures effectuées à température ambiante, on constate que des valeurs très voisines sont obtenues quelle que soit la méthode de croissance mise en œuvre (Tableau II). Si la vitesse limite des électrons atteint $10^{7} \mathrm{~cm} / \mathrm{s}$ à $300 \mathrm{~K}$, valeur très proche de celle des mêmes porteurs dans le germanium refroidi à $77 \mathrm{~K}$, il n'en est pas de même pour les trous, puisque pour des champs de $10^{4} \mathrm{~V} / \mathrm{cm}$ elle est dix fois plus faible. Cette vitesse réduite des trous influencera les propriétés de détection des spectromètres.

1.1.3 Durée de vie des porteurs. - Elle varie considérablement d'un échantillon à 1'autre suivant la perfection cristalline et la concentration d'impuretés. La valeur de $\tau$ est généralement déterminée par des mesures d'amplitude des signaux délivrés par les compteurs sous bombardement de particules chargées en employant la relation de Hecht [24]. Les durées de vie trouvées expérimentalement augmentent avec le carré du champ électrique sans, toutefois, atteindre la microseconde pour les champs les plus intenses. A titre purement indicatif, nous avons reporté sur le tableau III quelques valeurs récentes de $\mu \tau$. On notera qu'au cours des dernières années ces valeurs ne se sont pas sensiblement améliorées mais, ce qui constituait une performance il $y$ a quelque temps, est maintenant devenu routine dans plusieurs laboratoires.

\begin{tabular}{|c|c|c|c|}
\hline \multicolumn{4}{|c|}{ TABLEAU III } \\
\hline \multirow[b]{2}{*}{ Auteurs } & \multirow{2}{*}{$\begin{array}{l}\text { Méthode de } \\
\text { croissance }\end{array}$} & \multicolumn{2}{|c|}{$\mu \tau\left(\mathrm{cm}^{2} / \mathrm{V}\right)$} \\
\hline & & $e^{-}$ & $e^{+}$ \\
\hline 一 & 一 & - & - \\
\hline Bell [21] & THM (Cl) & $3 \times 10^{-3}$ & $3 \times 10^{-4}$ \\
\hline Zanio [38] & Epuisement & & \\
\hline & solution (In) & $6 \times 10^{-4}$ & $5 \times 10^{-6}$ \\
\hline Taguchi $[107]$ & THM & $4 \times 10^{-4}$ & $2 \times 10^{-5}$ \\
\hline Martin [108] & THM & $1,5 \times 20^{-3}$ & \\
\hline Alekseenko [110] & & $2,6 \times 10^{-3}$ & $4 \times 10^{-4}$ \\
\hline
\end{tabular}

TABLEAU II

\begin{tabular}{|c|c|c|c|c|}
\hline Auteurs & $\begin{array}{c}\text { Technique de } \\
\text { croissance }\end{array}$ & $\begin{array}{c}\text { Mobilité } \\
\mathrm{e}^{-}\end{array}$ & $\begin{array}{c}\left(\mathrm{cm}^{2} \cdot \mathrm{V} \cdot \mathrm{s}\right) \\
\mathrm{e}^{+}\end{array}$ & Méthode \\
\hline- & 一 & - & - & - \\
\hline Zanio & Bridgman & 1000 & 70 & Temps de vol (TV) \\
\hline Bell & THM & 1000 & 80 & TV \\
\hline Canali & Epuisement de solution & $750-1500$ & & SLLC \\
\hline Triboulet & Fusion de zone & 1100 & & Hall \\
\hline Taguchi & THM & 1100 & 94 & TV \\
\hline Martin & THM & 1000 & 80 & TV \\
\hline Nous & THM & 1000 & $70-80$ & $\mathrm{TV}$ \\
\hline
\end{tabular}


1.2 PrÉPARATION ET CARACTÉRISTIQUES DES DÉTECTEURS. — Les cristaux sont d'abord découpés en plaquettes d'épaisseur 1-4 mm qui sont nettoyées par les procédés conventionnels de la technologie des semiconducteurs. Les traitements ultérieurs dépendent de la nature du matériau :

- les cristaux de type $\mathrm{N}$ de faible résistivité sont décapés dans une solution de brome-méthanol dans une atmosphère inerte, puis après lavage et séchage, le contact redresseur est obtenu sur une partie monocristalline par dépôt d'or [25] ou par implantation ionique $[26,27]$;

- les cristaux de type $\mathbf{P}$ compensés par l'indium ou non dopés sont le plus souvent simplement polis avant le dépôt des contacts ( $\mathrm{Au}, \mathrm{Al}$, Aquadag);

- les cristaux de type $\mathrm{P}$ compensés au chlore doivent subir après le décapage chimique une oxydation superficielle pour former des structures MOS ou MIS (voir ci-dessous).

Des contacts dissymétriques sont parfois employés (Fig. 2) pour tirer le meilleur profit du produit $\mu \tau$ plus favorable des électrons [28].

1.3 COURANT DE FUITE. - Les courants de fuite sont compris entre $10^{-7}$ et $10^{-10}$ A pour des tensions de 100-1 000 volts suivant la nature des matériaux et l'importance des volumes sensibles.
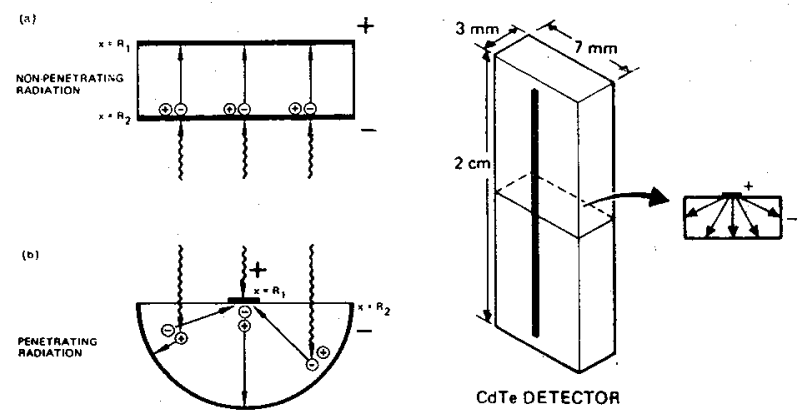

Fig. 2. - Structures de détecteurs CdTe favorisant la collecte des électrons.

1.4 CAPACITÉs. - Les capacités des échantillons compensés au chlore ou à l'indium sont indépendantes de la tension appliquée en raison de la résistivité élevée des substrats, mais elles dépendent de la fréquence du pont de mesure. Lorsque les surfaces sont décapées chimiquement, des capacités parasites importantes peuvent apparaître liées aux états de surface [29].

Les matériaux de plus faible résistivité conduisent à une loi de la variation avec la tension de la forme $C \alpha V^{-n}$ avec $n \approx 0,2-0,3$. Ce comportement particulier n'est pas dû aux effets de surface comme

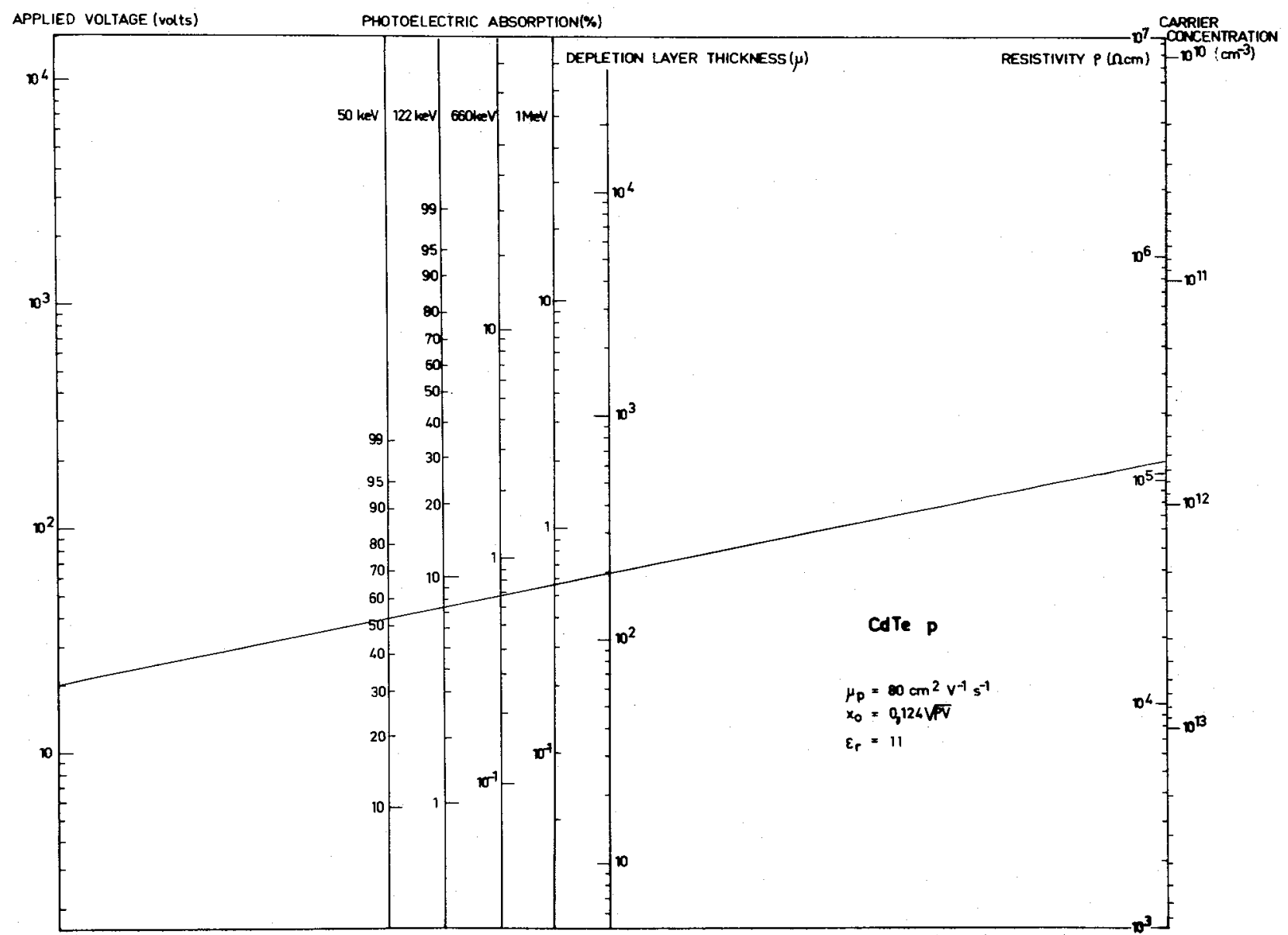

Fíg. 3. - Abaque permettant de déterminer l'extension de la zone désertée dans CdTe de type P en fonction de la résistivité du matériau et de la tension appliquée. 
indiqué dans la littérature [30-33] mais résulte de niveaux profonds existant dans le matériau [34].

1.5 VOLUMES SENSIBLES. - L'épaisseur $X$ de la zone sensible d'un compteur est directement proportionnelle à la résistivité $\rho$ et à la tension appliquée suivant les relations :

$$
\begin{aligned}
& X=0,12 \sqrt{\rho V} \quad \text { pour les cristaux de type P } \\
& (\mu)(\Omega . \mathrm{cm}, \text { volts })
\end{aligned}
$$$$
X=0,4 \sqrt{\rho V} \quad \text { pour les cristaux de type } \mathrm{N} \text {. }
$$

L'abaque de la figure 3 permet de calculer $X$ pour les cristaux de type $P$. Plusieurs auteurs $[35,36]$ ont pu vérifier que $X$ croît bien avec $V^{1 / 2}$, par contre, la résistivité réelle $\rho^{\prime}$ est en fait bien inférieure aux valeurs énoncées plus haut. Des essais comparatifs effectués sur un grand nombre d'échantillons ont permis de montrer que $\rho^{\prime}$ ne dépasse que très rarement $10^{6} \Omega . \mathrm{cm}$ (Fig. 4), soit $500-1000$ fois moins que la valeur escomptée [37]. De ce fait, les épaisseurs sensibles réelles ne dépassent pas quelques $\mathrm{mm}$ et ce n'est que tout récemment que $6 \mathrm{~mm}$ d'épaisseur sensible ont pu être atteints [37]. Les surfaces actives des spectromètres sont de l'ordre de 0,1 à $0,3 \mathrm{~cm}^{2}$ le plus souvent, bien que des aires de $2 \mathrm{~cm}^{2}$ aient été atteintes [38]. Plusieurs solutions ont été envisagées pour augmenter les volumes sensibles :

- mosaïques de plusieurs détecteurs (Fig. 5) [38] ; - empilement de plusieurs compteurs [39].

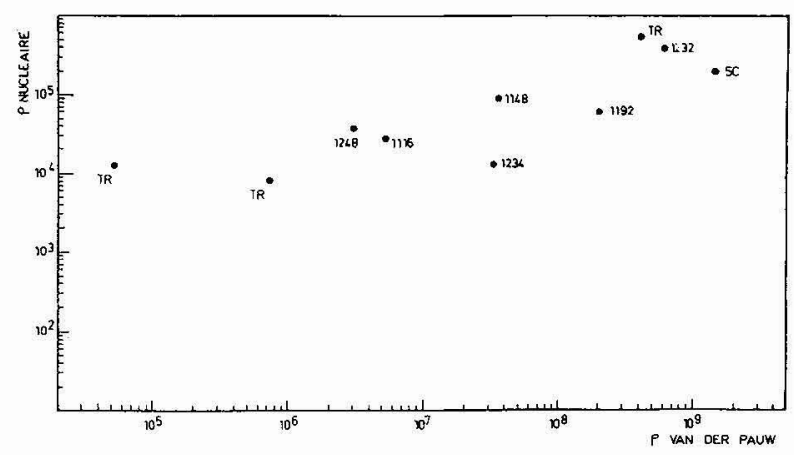

FIG. 4. - Comparaison des résistivités mesurées par la technique van der Pauw et déduite de mesures nucléaires pour divers cristaux.

1.6 CARACTÉRISTIQUES DE DÉTECTION. - Quatre caractéristiques nous ont paru essentielles, nous les analyserons successivement :

1.6.1 Hauteur d'impulsion. - Les énergies requises pour créer une paire électron-trou étant respectivement de $3,61 \mathrm{eV}(300 \mathrm{~K})$ dans le silicium, de $2,98 \mathrm{eV}(77 \mathrm{~K})$ dans le germanium et de 4,46 eV (300 K) [41-43] dans le tellurure de cadmium les hauteurs d'impulsions issues de ce matériau ne correspondent dans le cas idéal qu'à $81 \%$ et $67 \%$ de celles d'un spectromètre $\mathrm{Si}$ et $\mathrm{Ge}$, respectivement, pour des rayonnements de même énergie. En fait, ces amplitudes ne seront observées que pour les

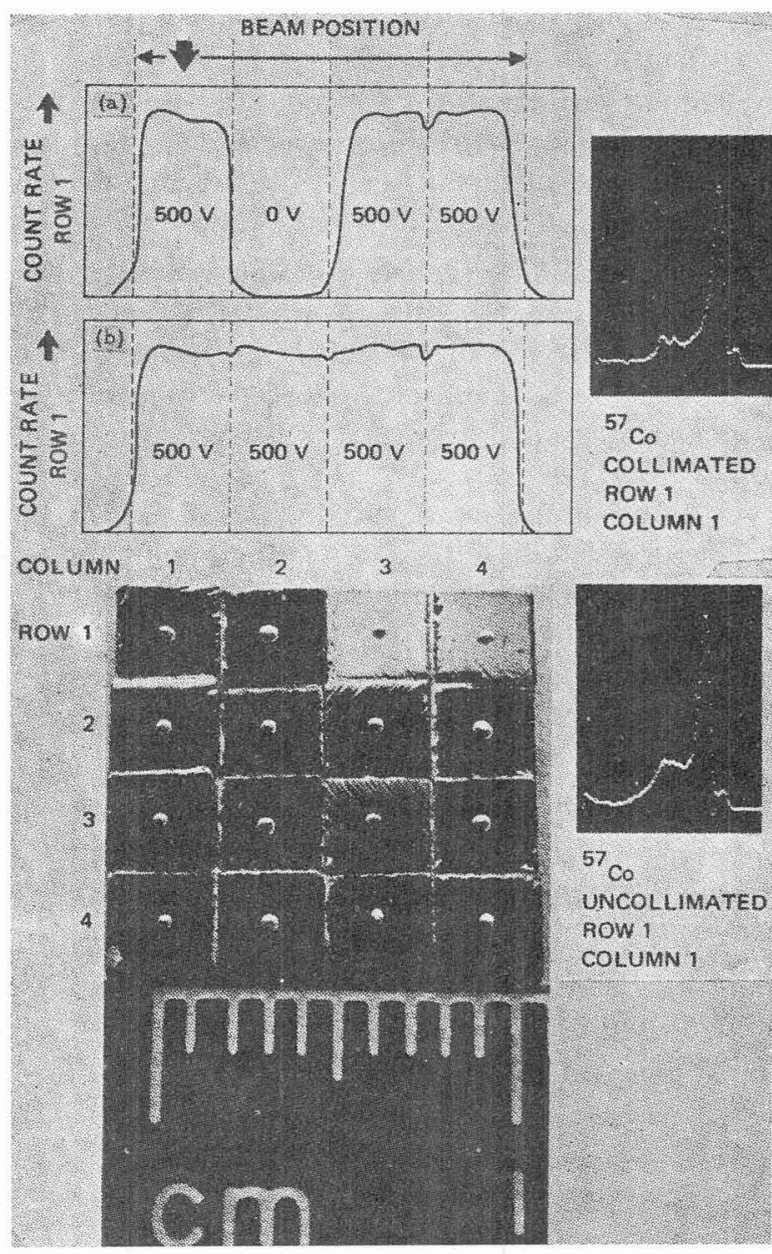

FIG. 5. - Exemple d'association de plusieurs détecteurs CdTe [38]

cristaux les plus purs, alors que dans la plupart des compteurs la collecte des charges ne correspondra qu'à 80-85 \% de l'amplitude théorique, par suite de phénomènes de capture sur des niveaux pièges.

1.6.2 Efficacité de détection. Cas idéal. - En spectrométrie $\gamma(E<1,5 \mathrm{MeV})$ seule l'efficacité photoélectrique ou l'absorption Compton conduisant au transfert total de l'énergie du photon au milieu absorbant est intéressante. A volume égal le gain en efficacité sera de l'ordre de 5 par rapport au germanium (Fig. 6) pour une énergie de l'ordre de $500 \mathrm{keV}$. On notera (Fig. 7) que lorsque l'énergie croît, l'efficacité de détection diminue très rapidement par suite des épaisseurs sensibles $X$ encore trop faibles à l'heure actuelle. D'où la nécessité de connaître avec précision ces dernières.

Trois moyens simples permettent d'obtenir $X$ :

- mesure de l'efficacité de détection pour des particules peu pénétrantes arrivant respectivement par l'électrode positive et négative en fonction de la tension. Lorsque les taux de comptage seront identiques toute l'épaisseur de la plaquette sera sensible ;

- mesure de l'efficacité de détection en fonction de la tension pour des photons énergétiques jusqu'à obtention d'un palier; 


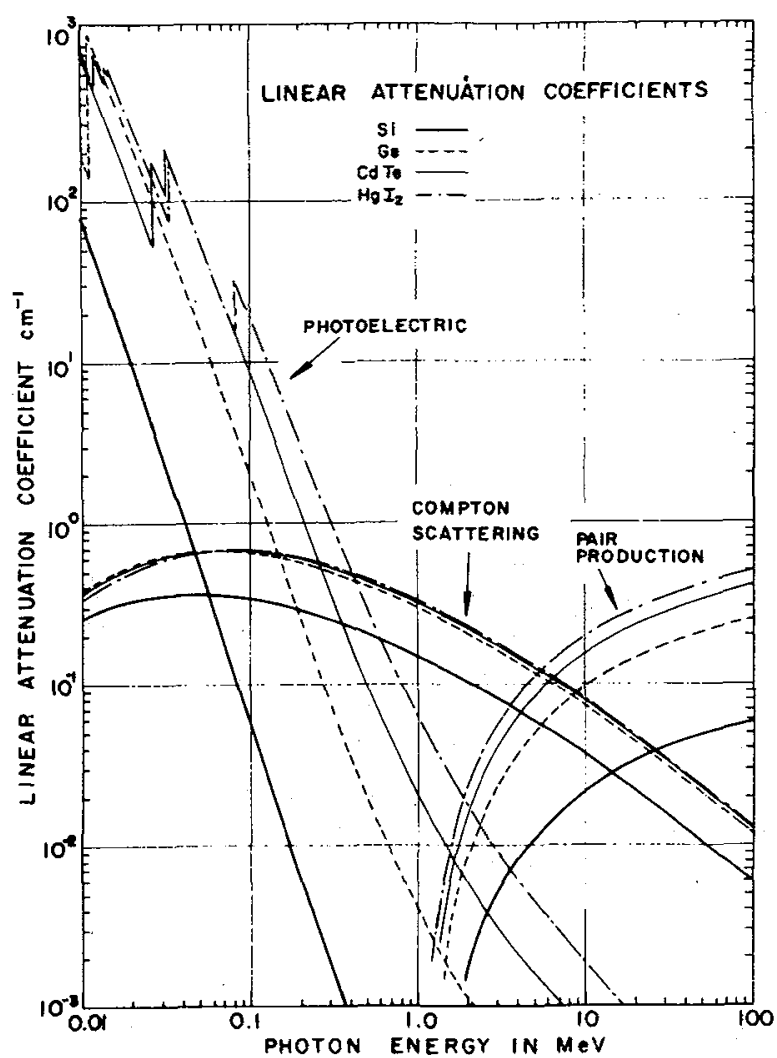

FIG. 6. - Coefficient d'absorption des photons en fonction de l'énergie dans le silicium, le germanium, le tellurure de cadmium et l'iodure mercurique.

- évaluation de l'importance relative du pic d'échappement par rapport au pic photoélectrique (voir ci-dessous).

- Phénomènes parasites. - Plusieurs paramètres peuvent dégrader l'efficacité de détection : échappement de rayons $\mathrm{X}$ : en raison des faibles volumes sensibles il existe une probabilité non négligeable de création de rayons $\mathrm{X}$ de fluorescence provenant de l'excitation par les rayons $\gamma$ des atomes de cadmium et de tellure. Ceci se traduit par des pics d'échappement se situant à des énergies $E_{\gamma}-E_{\mathrm{X}}$; $\left(E_{\mathrm{X}}(\mathrm{Cd})=23 \mathrm{keV}, \quad E_{\mathrm{X}}(\mathrm{Te})=27 \mathrm{keV} \ldots\right)$. Plusieurs auteurs ont analysé ces phénomènes $[44,45]$. L'influence des pics d'échappement décroît très vite avec l'énergie et l'épaisseur sensible. Cet effet peut alors être mis à profit pour mesurer avec précision l'épaisseur désertée, ou la résistivité effective [37] (Fig. 8).

- Piégeage des porteurs. - Les niveaux localisés dans la bande interdite peuvent retenir les porteurs en cours de transit vers les électrodes pendant un temps $\theta$ donné par $\theta^{-1}=\tau v_{\mathrm{th}} N_{\mathrm{T}}(\tau$ : section efficace de capture par un niveau piège de concentration $N_{\mathrm{T}}$, $v_{\mathrm{th}}$ : vitesse thermique des porteurs). Si le temps de capture est supérieur aux constantes de mise en forme de l'amplificateur, certains porteurs ne participeront plus à la formation du signal. A température ambiante, les niveaux situés plus profondément que $0,25 \mathrm{eV}$ seront les plus gênants pour la détection
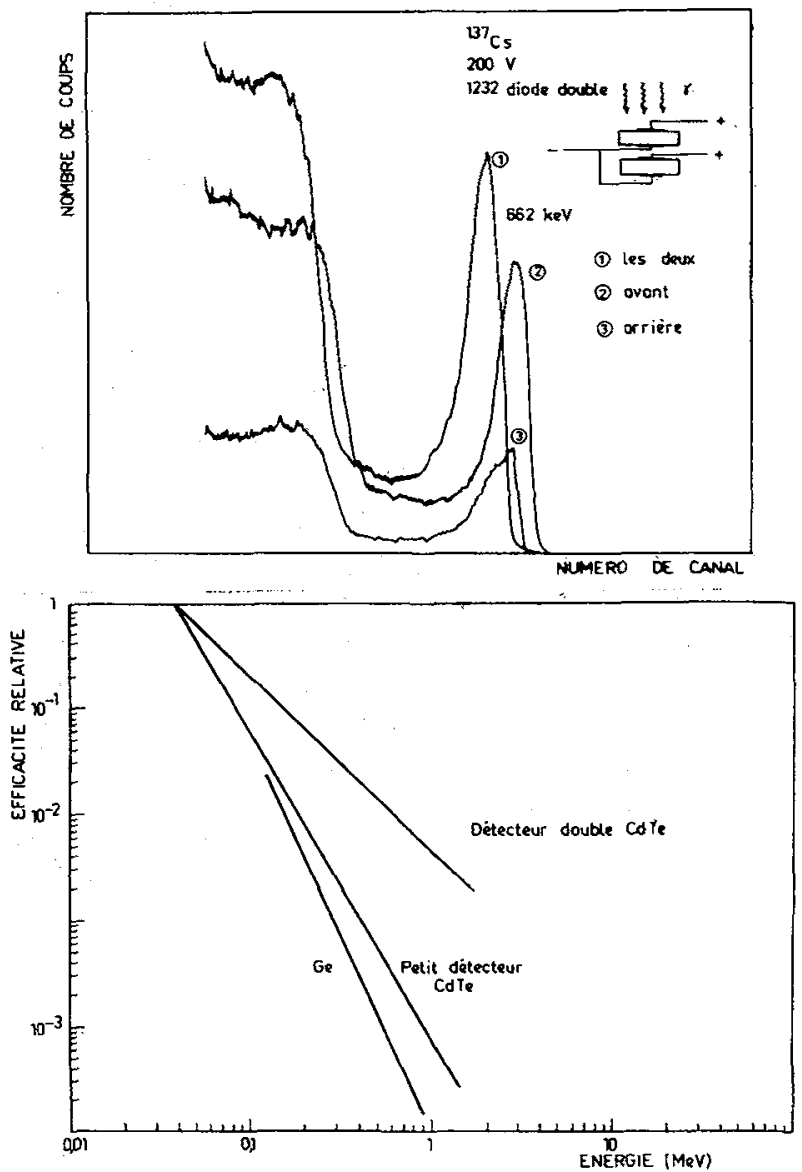

Fig. 7. - La partie supérieure de cette figure montre le spectre d'une source de ${ }^{137} \mathrm{Cs}$ enregistré à l'aide de deux diodes montées comme indiqué. La partie inférieure de cette figure représente l'efficacité relative de détection photoélectrique pour un détecteur Ge et CdTe de même volume ainsi que pour la diode double représentée en haut.

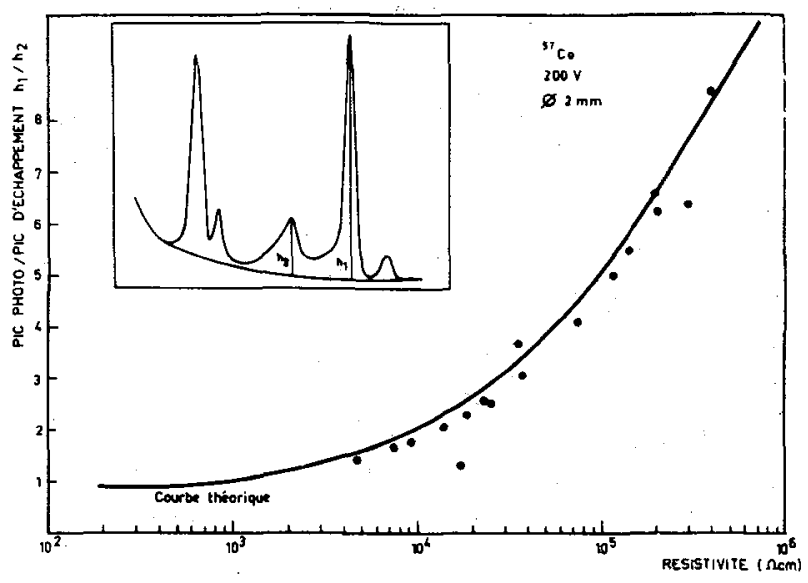

FIG. 8. - Evolution du rapport du pic photoélectrique au pic d'échappement pour une diode polarisée à $200 \mathrm{~V}$ en fonction de la résistivité apparente du matériau. La courbe en traits pleins est calculée, les points représentent des résultats expérimentaux.

nucléaire. Ces problèmes ont fait l'objet d'études approfondies par plusieurs groupes [46-48].

- Polarisation. - Ce phénomène sera étudié dans la partie consacrée à la stabilité des détecteurs.

1.6.3 Résolution en énergie. - Sources d'élargissement : il est bien connu que la largeur à mi-hauteur 
d'un pic d'absorption totale obtenu à l'aide d'un détecteur à semiconducteur est la résultante de plusieurs contributions :

- Fluctuations statistiques de création de porteurs. - Bien que le facteur de corrélation entre collisions successives ne soit pas établi expérimentalement à l'heure actuelle, on peut penser, à la lumière de considérations théoriques [49] que sa valeur sera très faible $(0,04)$, par conséquent l'élargissement résultant de ces fluctuations pourra être négligé devant les autres contributions.

- Bruit de l'ensemble détecteur-amplificateur. Les fluctuations du courant de fuite du détecteur, le bruit des éléments du préamplificateur amènent un élargissement des raies. En supposant que les constantes de temps d'intégration et de différentiation de l'amplificateur sont les mêmes, on peut calculer aisément l'élargissement produit par le courant de fuite (Fig. 9). On remarquera que très souvent pour obtenir une contribution minimale à l'élargissement de cet effet il convient d'employer des constantes de temps brèves, or dans ce cas, la collection complète des charges ne pourra se faire et il faudra trouver un compromis qui se situe aux alentours de $1 \mu \mathrm{s}$.

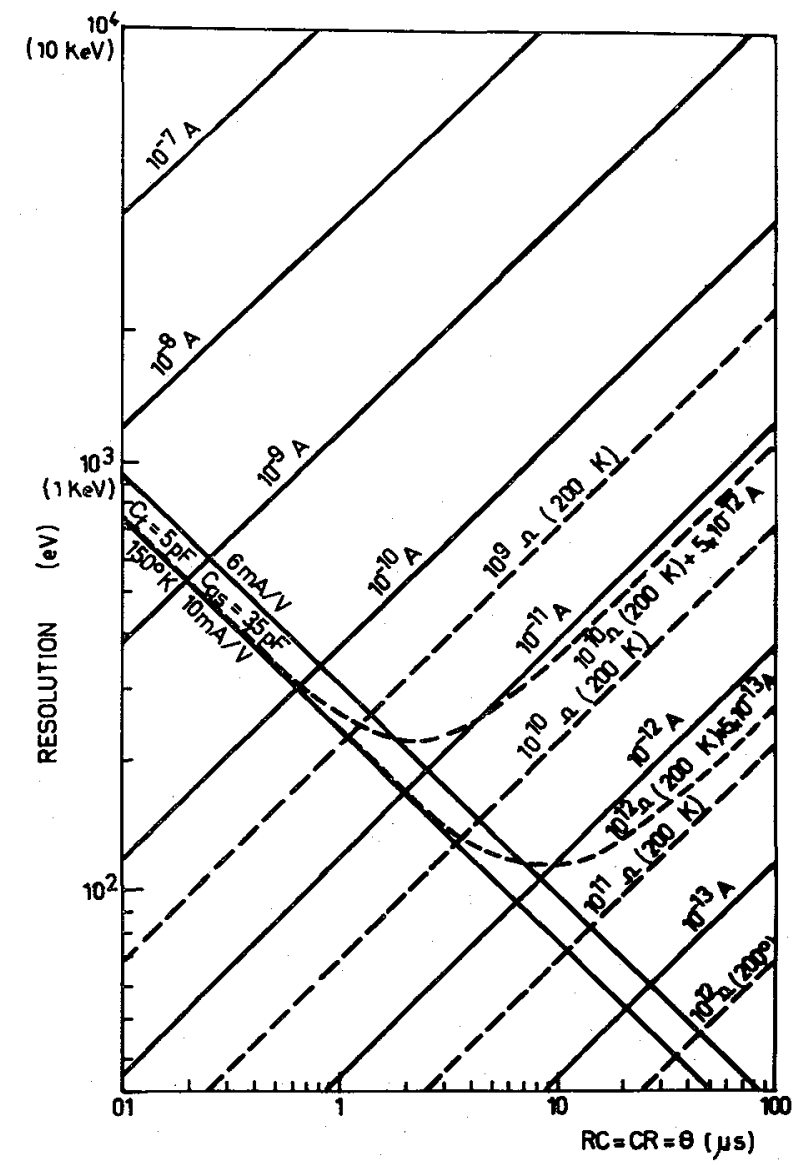

FIG. 9. - Abaque permettant de calculer la résolution en énergie en fonction des constantes de temps d'intégration et de différenciation (identiques) de l'amplificateur pour diverses valeurs du courant de fuite du détecteur CdTe.
- Défaut balistique. - Lorsque l'épaisseur sensible croît, le temps de montée des impulsions s'allonge, de sorte que pour les constantes de temps de mise en forme de l'amplificateur généralement utilisées, les signaux ne peuvent atteindre leur amplitude. Toutefois, un ensemble électronique vient d'être proposé qui permettra de réduire notablement les effets de ce défaut balistique [50].

- Fluctuations liées au piégeage, défaut de collection. - De nombreux auteurs ont étudié l'aspect théorique de ce problème [51-54] essentiellement pour les compteurs $\mathrm{Si}$ et $\mathrm{Ge}$. Dans le cas du tellurure de cadmium une collection incomplète $(1-\eta)$ se traduit par un élargissement de la forme :

$$
L_{\mathrm{t}} \approx K(1-\eta) E^{1 / 2} \text {. }
$$

Lorsque le photoélectron a une énergie suffisante il peut s'échapper hors du volume sensible ce qui se traduit par un élargissement notable.

- Résultats expérimentaux. - Nous avons rassemblé sur le tableau IV quelques résultats expérimentaux tirés de la littérature. Ces valeurs s'améliorent quelque peu pour les compteurs de faible volume lorsque les températures de fonctionnement sont ramenées à $-20-50^{\circ} \mathrm{C}$. On notera une dégradation rapide de la résolution lorsque l'énergie croît (échappement des photoélectrons). La détérioration de la résolution observée pour les volumes les plus importants est due à l'accroissement du courant de fuite ainsi qu'à l'inhomogénéité des cristaux actuels.

\begin{tabular}{|c|c|c|c|}
\hline \multirow{4}{*}{ Energie $(\mathrm{keV})$} & \multicolumn{3}{|c|}{ TABLEAU IV } \\
\hline & \multicolumn{3}{|c|}{ Résolution (keV) } \\
\hline & & Volumes & \\
\hline & $0,3 \mathrm{~mm}^{3}$ & $5-10 \mathrm{~mm}^{3}$ & $350-400 \mathrm{~mm}^{3}$ \\
\hline $6\left({ }^{55} \mathrm{Fe}\right)$ & 1,1 & & \\
\hline $27\left({ }^{125} 1\right)$ & & & 9,2 \\
\hline $60\left({ }^{241} \mathrm{Am}\right)$ & 1,7 & 4,1 & 12 \\
\hline $122\left({ }^{57} \mathrm{Co}\right)$ & volume & 4,5 & \\
\hline $661\left({ }^{137} \mathrm{Cs}\right)$ & trop faible & 8 & 40 \\
\hline
\end{tabular}

1.6.4 Stabilité des détecteurs. - Le vieillissement des compteurs n'a pas fait l'objet jusqu'ici d'une étude systématique. Il semble, toutefois, que celui-ci ne donne pas lieu à des dégradations notables des performances. Par contre, la stabilité sur des intervalles de temps courts (polarisation) a fait l'objet de plusieurs travaux.

- Phénomènes de polarisation. - Il est connu depuis fort longtemps qu'une polarisation apparaît dans les isolants parcourus par des courants très faibles. Les premiers détecteurs nucléaires solides ne purent s'imposer (vers 1950) par suite de la diminution progressive de l'amplitude des signaux et de l'efficacité de comptage en fonction du temps d'irradiation [55]. La variation des propriétés de détection résulte de l'augmentation progressive du nombre de porteurs piégés dans le matériau qui transforme le champ électrique d'une structure de type N-i-P à celui d'une structure N-P (Fig. 10). Différents modèles ont été récemment proposés 


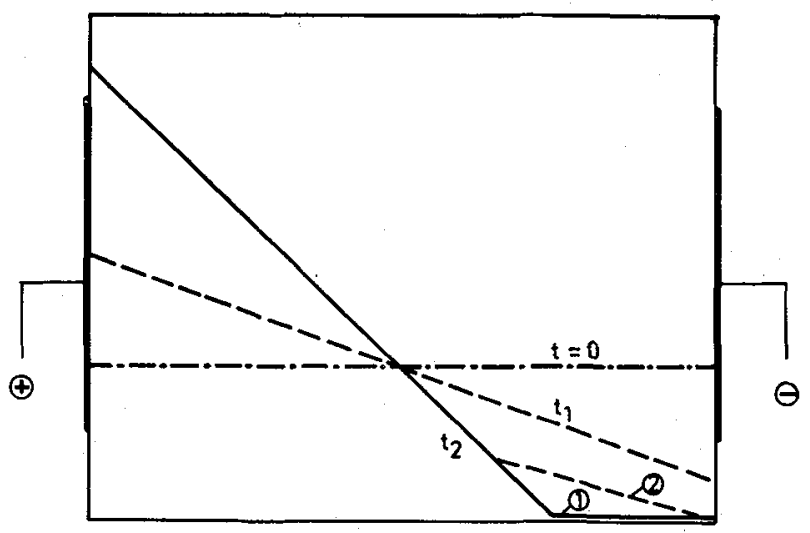

FIG. 10. - Evolution de la distribution du champ électrique dans un détecteur en fonction du temps en présence de polarisation.

pour expliquer cet effet [56]. On a pu montrer [57] que la réalisation d'une structure MOS ou MIS en surface supprime la polarisation.

- Stabilité sous irradiation. - Très peu de mesures ont été effectuées à ce jour [58-60]. En général, on observe tout d'abord une amélioration des performances suivie d'une dégradation notable pour des flux de rayonnements $\gamma\left({ }^{60} \mathrm{Co}\right)$ supérieurs à $2 \times 10^{5} \mathrm{rad} / \mathrm{h}$. Un recuit à $100-200^{\circ} \mathrm{C}$ permet de rétablir la situation initiale.

2. Iodure mercurique. - Ce matériau a été étudié depuis plus de vingt ans tant en France qu'à l'étranger pour ses propriétés photoconductrices ou excitoniques [61-65]. Dès 1955 on avait constaté [66] que $\mathrm{HgI}_{2}$ était sensible aux rayons $X$, pourtant ce n'est qu'en 1971 que le premier spectromètre mettant en cuvre ce matériau a vu le jour [67]. Certaines caractéristiques physiques rendent ce matériau particulièrement attractif pour la spectrométrie $y$ ou $\mathrm{X}$.

C'est un semiconducteur de structure lamellaire de couleur rouge, pouvant être clivé aisément. Ses principales propriétés physiques sont résumées dans la référence [68].

La croissance s'effectue à basse température $\left(<127^{\circ} \mathrm{C}\right)$ soit à partir d'un solvant, ce qui produit directement des plaquettes minces, soit par transport en phase gazeuse. Des monocristaux dont le poids atteint $200 \mathrm{~g}$ ont été préparés par ce dernier procédé.
2.1 CARACTÉRISTIQUES DES MATÉRIAUX. 2.1.1 Résistivité. - La largeur de bande $(2,1 \mathrm{eV})$ élevée de $\mathrm{HgI}_{2}$ conduit à des résistivités très élevées sans compensation extérieure. Dans les cristaux les plus purs seuls quelques niveaux pièges ont été trouvés et la stœchiométrie semble pouvoir être ajustée convenablement en employant lors de la croissance un excès d'iode [69].

2.1.2 Mobilité et vitesse des porteurs. - Nous avons rassemblé sur le tableau $\mathrm{V}$ les valeurs de mobilité et les vitesses limites des porteurs reportées dans la littérature. Il apparaît que la mobilité des électrons est de 100 et celle des trous de $4 \mathrm{~cm}^{2} / \mathrm{V}$.s. jusqu'à des champs de $10^{5} \mathrm{~V} / \mathrm{cm}$. Il semble que ces valeurs constituent les mobilités intrinsèques non limitées par les défauts ou les impuretés. Dans ces conditions la collecte des trous sera délicate et limitera l'épaisseur sensible des compteurs à $1 \mathrm{~mm}$ environ.

2.1.3 Durée de vie des porteurs. - Comme on peut le constater sur la figure 11 , la durée de vie $\tau$ des trous est particulièrement courte. Celle des électrons comme celle des trous varie très fortement avec le champ électrique indiquant que des phénomènes de piégeage limitent $\tau$. L'origine des centres responsables de cet effet n'est pas établie actuellement.

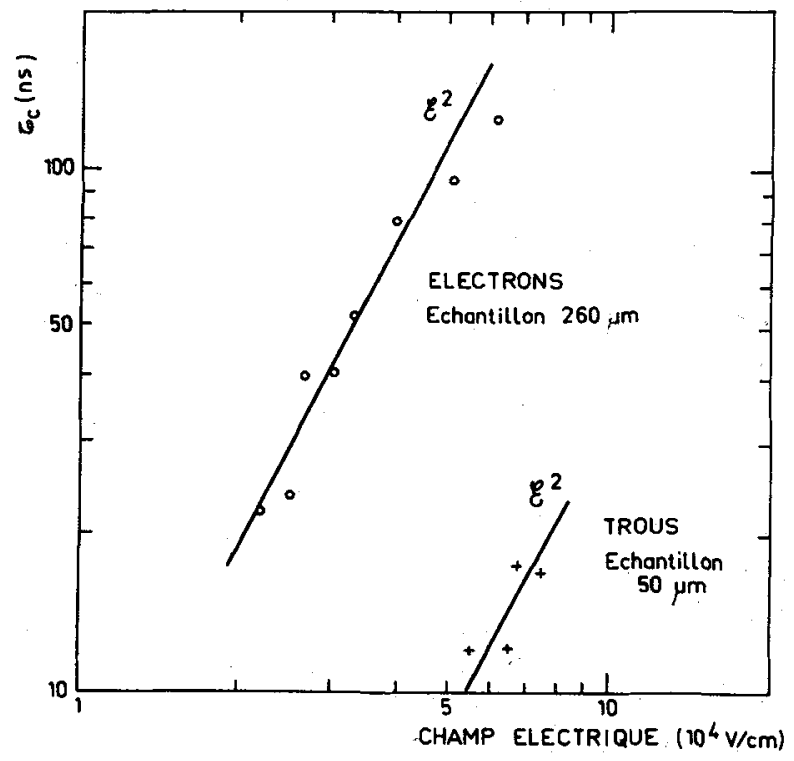

FIG. 11. - Durée de vie des électrons et des trous dans $\mathrm{HgI}_{2}$ en fonction du champ électrique.

\section{TABLEAU V}

\begin{tabular}{lc}
\multicolumn{1}{c}{ Auteurs } & $\mu_{\text {ell }}\left(\mathrm{cm}^{2} \cdot \mathrm{V} . \mathrm{s}\right)$ \\
Malm & -70 \\
Swierkowski & $3-94$ \\
Llacer & 120 \\
Martin & $55-75$ \\
Ponpon & 100 \\
Ottaviani & 100 \\
Schieber & 80
\end{tabular}

Mobilité-vitesse $(300 \mathrm{~K})$

\begin{tabular}{ccc}
\multicolumn{2}{c}{ Mobilité-vitesse $(300 \mathrm{~K})$} & \\
$\mu_{\mathrm{e} \perp}\left(\mathrm{cm}^{2} . \mathrm{V} . \mathrm{s}\right)$ & $V_{\text {saturation }}(\mathrm{cm} / \mathrm{s})$ & $\mu_{\mathrm{h} \|}$ \\
& $6,5 \times 10^{5}\left(\mathrm{champ} 10^{4} \mathrm{~V} / \mathrm{cm}\right)$ & 4 \\
& & 3 \\
& $6 \times 10^{6}(80 \mathrm{kV} / \mathrm{cm})$ & 15 \\
65 & $6 \times 10^{6}(60 \mathrm{kV} / \mathrm{cm})$ & $3-4$ \\
& & 4 \\
& & 4
\end{tabular}


2.2 PRÉPARATION DES DÉTECTEURS. - Alors que les plaquettes monocristallines obtenues par la technique de croissance en solvant peuvent être employées directement, les matériaux massifs doivent être découpés ou clivés en plaquettes d'épaisseur 200-1 $000 \mu$. Les dommages consécutifs à ces opérations sont enlevés par un décapage chimique superficiel. Les contacts sont réalisés sur les faces 001 parallèles au plan de clivage. Les matériaux formant les contacts doivent être chimiquement inertes. En pratique, on emploie soit une peinture d'une émulsion de carbone (genre aquadag) soit le dépôt, par évaporation sous vide, de carbone, palladium ou germanium. Des surfaces de $1 \mathrm{~cm}^{2}$ ont été réalisées. Le matériau étant très volatil, il est nécessaire, pour assurer une stabilisation à long terme, d'enrober le détecteur dans une résine plastique.

Le courant de fuite est très faible, généralement inférieur à $10^{-10} \mathrm{~A} / \mathrm{cm}^{2}(300 \mathrm{~K})$ pour des champs électriques de l'ordre de $1 \mathrm{kV} / \mathrm{cm}$. Ces valeurs sont nettement inférieures à celles des spectromètres CdTe.

2.3 CARACTÉRISTIQUeS DE DÉTECTION. 2.3.1 Energie de création d'une paire électron-trou. - Pour les cristaux de structure cubique on avait établi qu'il existe une relation entre cette énergie $\varepsilon$ et la largeur de bande $E g$ de la forme $(300 \mathrm{~K})$ :

\section{(eV)}

$$
\varepsilon=1,95 \mathrm{Eg}+1,4
$$

Compte tenu de la largeur de bande de $\mathrm{HgI}_{2}$ on s'attend à trouver $\varepsilon=5,5 \mathrm{eV}$. En réalité, plusieurs auteurs $[70,71]$ indiquent une valeur nettement plus faible pour ce cristal de structure lamellaire, de l'ordre de 4,2 eV. Ce résultat a deux conséquences intéressantes :

- pour un photon d'énergie donnée, l'amplitude du signal est de $30 \%$ supérieure à celle prévue, d'où un meilleur rapport signal à bruit ;

- des fluctuations statistiques réduites, d'où une meilleure résolution en énergie.

2.3.2 Résolution en énergie. - Le matériau étant transparent à la lumière visible, il est possible de sélectionner les meilleures zones de cristaux exempts notamment d'anneaux de dislocations [7274]. Grâce au faible bruit de fond et à l'énergie réduite nécessaire à la création d'une paire électrontrou, il est possible d'effectuer la spectrométrie de rayons $X$ (même mous) et $\gamma$. Nous avons rassemblé sur le tableau VI quelques résultats atteints récemment.

Les figures 12 à 14 représentent les meilleurs spectres publiés dans la littérature à diverses énergies [75, 76]. En examinant ces résultats on constate que :

- des résolutions très intéressantes peuvent être atteintes à température ambiante. Il est très probable que ces compteurs seront amenés à remplacer les spectromètres $\mathrm{Si}(\mathrm{Li})$ dans les ensembles d'analyse par fluorescence $X$;

- la dégradation rapide de la résolution aux énergies élevées. Comme pour le tellurure de cadmium cela résulte de l'échappement partiel des photoélectrons hors du volume sensible. L'épaisseur de ce dernier ne peut être accrue au-delà de $1 \mathrm{~mm}$ à cause de la vitesse de déplacement faible des trous;

- la présence de pics d'échappement, résultant des numéros atomiques élevés des constituants du matériau.

2.3.3 Efficacité de détection. - Plusieurs auteurs $[71,77]$ ont calculé la forme des raies spectrales en fonction de divers paramètres tant pour des structures planes qu'hémisphériques. Leurs résultats montrent l'intérêt d'accroître $\mu \tau$ et l'épaisseur $X$. Malheureusement, ce dernier paramètre ne peut être augmenté pour les raisons déjà

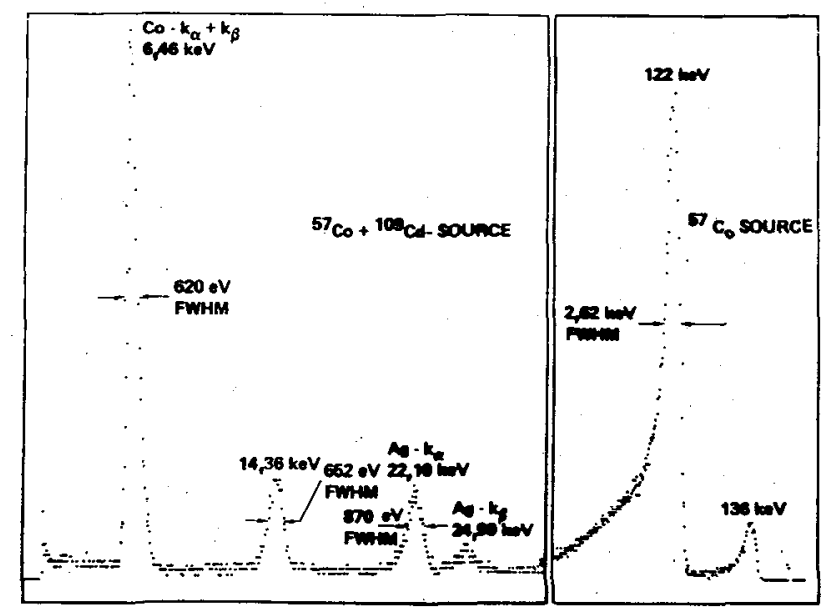

FIG. 12. - Spectre $\mathrm{X}$ et $\gamma$ enregistré par un détecteur $\mathrm{HgI}_{2}$ bombardé par ${ }^{57} \mathrm{Co}$ et ${ }^{109} \mathrm{Cd}[75]$.

TABLEAU VI

Résolution (300 K)

Energie

(keV)

1,5

5,9

60

122

661

$12 \mathrm{~mm}^{2} \times 0,4 \mathrm{~mm}$

$12 \mathrm{~mm}^{2} \times 0,4 \mathrm{~mm}$

$100 \mathrm{~mm}^{2} \times 0,5 \mathrm{~mm}$

$1,1 \mathrm{keV}$

$2,5 \mathrm{keV}$

$2,6 \mathrm{keV}$

$4,5 \mathrm{keV}$ 


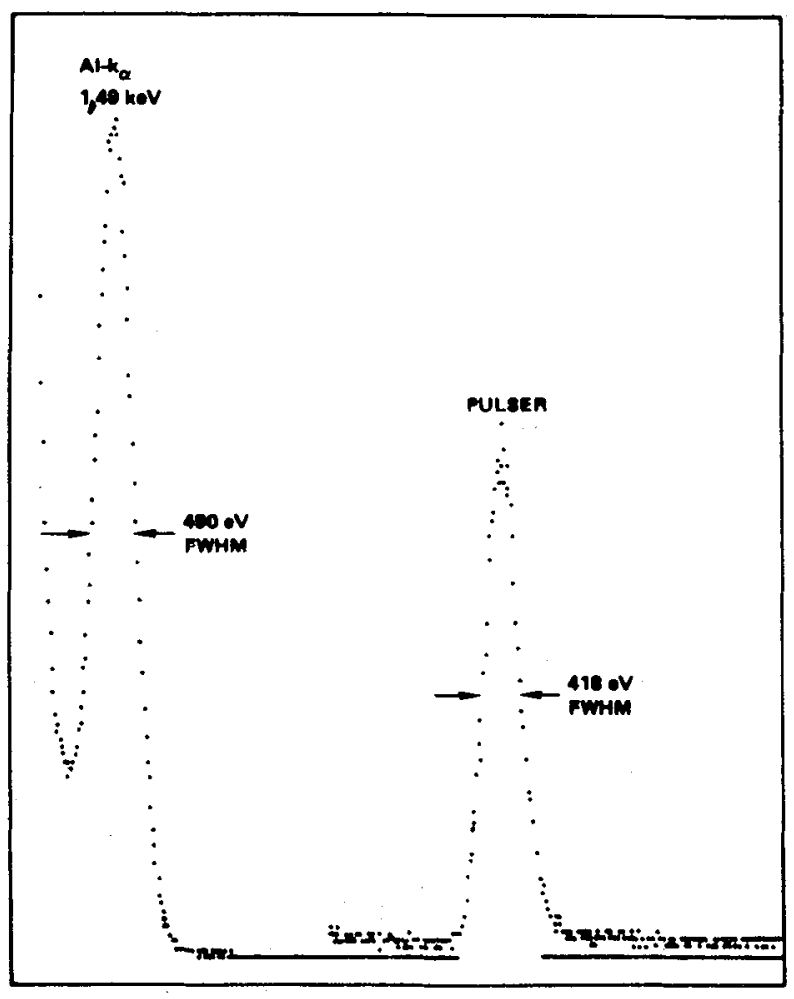

FIG. 13. - Rayons $X$ de l'aluminium enregistrés à l'aide d'un compteur $\mathrm{HgI}_{2}[75]$.
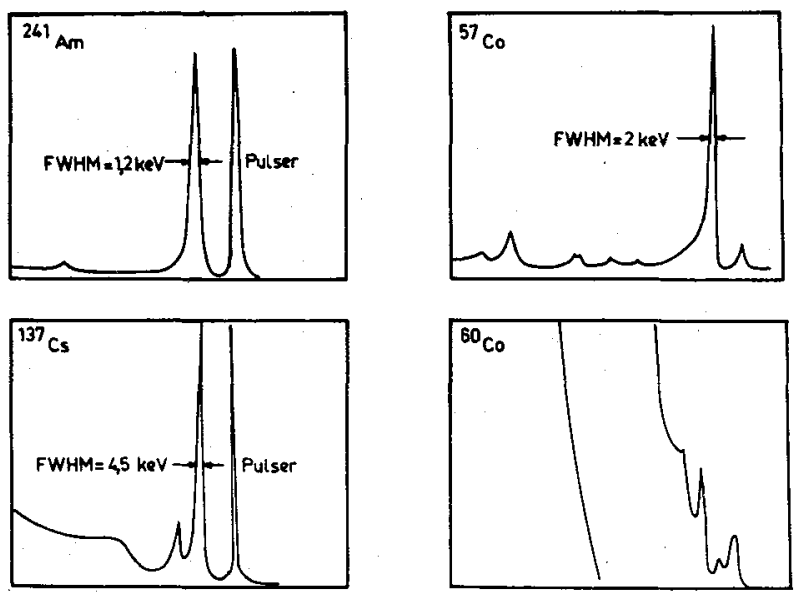

FIG, 14. - Divers spectres de rayons $\gamma$ enregistrés à l'aide d'un compteur $\mathrm{HgI}_{2}[76]$.

indiquées. Quelques résultats sont reportés sur les figures 15 et 16 . Des associations de 12 compteurs de $1 \mathrm{~cm}^{2}$ ont été décrites dans la littérature [73] afin d'accrô̂tre les efficacités de détection.

Mentionnons également que des phénomènes de polarisation ont été observés dans certains cristaux.

A la lumière de ces résultats il nous semble que les spectromètres à $Z$ élevés capables de fonctionner à température ambiante sont en bonne voie de supplanter d'ici quelques années les spectromètres Ge ou Si de faible volume. Par contre, les compteurs Ge

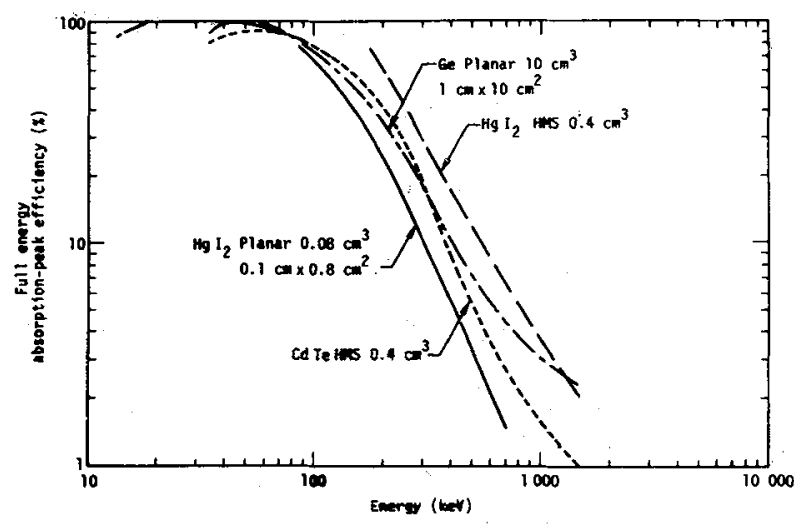

FIG. 15. - Efficacité calculée de compteurs $\mathrm{Ge}$, CdTe et $\mathbf{H g I}_{2}$ en fonction de l'énergie des photons $\gamma$.

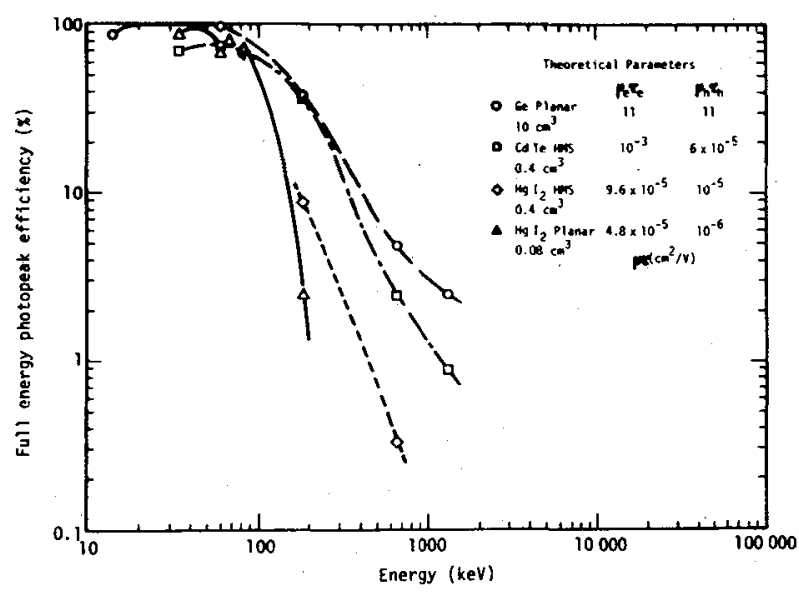

FiG. 16. - Efficacité calculée de compteurs Ge, CdTe et $\mathrm{HgI}_{2}$ en fonction de l'énergie des photons $\gamma$ en tenant compte des valeurs finies de $\mu \tau$.

ne pourront être remplacés lorsque 2 propriétés sont requises : grands volumes sensibles, détection de photons d'énergie élevée.

2.4 SPECTROMÉTRIE D'IONS LOURDS. - Le développement rapide des études de physique nucléaire auprès des accélérateurs à ions lourds a sensiblement compliqué les problèmes de détection des produits de réaction puisqu'il s'agit non seulement de connaître les énergies de ces particules mais encore d'identifier les fragments formés. De ce fait l'emploi de télescopes $\Delta E, E$, des techniques de temps de vol s'est généralisé au cours des dernières années. Les ensembles de détection font appel aux détecteurs à feuilles (électrons secondaires) à gaz (compteur proportionnel, à grilles), à scintillations ainsi qu'aux dispositifs semiconducteurs. En nous limitant à ces derniers, il apparaît que deux types de problèmes se posent : la réalisation de compteurs $\Delta E$ très minces, la détection de l'énergie totale $E$ des produits de réaction.

3. Compteurs minces $\Delta \boldsymbol{E}$. - Jusqu'à une période récente, les diodes minces $\Delta E$ à base de silicium 
étaient obtenues à partir de plaquettes de silicium qui étaient d'abord amincies par rôdage mécanique puis décapées chimiquement. Ces dispositifs présentaient deux inconvénients majeurs : d'une part ils étaient très fragiles pour des épaisseurs inférieures à $25 \mu$, d'autre part il était difficile de préparer des compteurs d'épaisseur uniforme.

3.1 PRÉparation Des COMPTEURS. - En 1973 [78], nous avons proposé une voie toute différente pour réaliser ces dispositifs en partant de plaquettes de silicium épitaxiales qui sont largement employées dans l'industrie des composants électroniques actifs. Rappelons que la croissance de films épitaxiés de silicium s'effectue généralement par dépôt sur un substrat également de silicium, mais de faible résistivité, d'un film de silicium à partir d'une phase vapeur contenant également le dopant. Contrairement aux techniques de diffusion thermique des couches très uniformes tant en épaisseur que dans la distribution des dopants peuvent être produites. Ce procédé étant industriel, les plaquettes seront d'un coût raisonnable. Les propriétés essentielles de ces couches, qui nous intéressent ici, sont rassemblées sur le tableau VII.

\section{TABLEAU VII}

\section{Silicium épitaxial}

$\begin{array}{ll}\text { Type } & \mathrm{N} \text { et } \mathrm{P} \\ \text { Résistivité } & 10^{-2} \text { à } 10^{2} \Omega . \mathrm{cm} \\ \text { Epaisseur } & 1-100 \mu \\ \text { Variation d'épaisseur } / \mathrm{cm}^{2} & <1000 \AA \\ \text { Diamètre possible } & 10 \mathrm{~cm} \\ \text { Substrat } & \text { le plus souvent silicium } \\ \text { Défauts à surveiller } & \text { défauts d'empilement } \\ & \text { (" stacking faults») }\end{array}$

Par ailleurs, il est connu [79] que la vitesse de dissolution anodique du silicium dans un bain convenable peut dépendre considérablement de la résistivité du matériau. Par conséquent, en choisissant des couches de résistivité élevée déposées sur des substrats de faible résistivité, il sera possible de trouver des conditions telles que le substrat soit dissous sur une aire choisie, alors que l'attaque cessera dès que le film sera atteint. Il ne restera qu'à former une diode Schottky ou une jonction $\mathrm{P}-\mathrm{N}$ pour terminer le compteur. Les principales étapes d'élaboration d'un tel dispositif sont schématisées sur la figure 17.

Dès à présent, plusieurs variantes à ce type de compteurs peuvent être envisagées (Fig. 18) :

- Diode à barrière de surface ou à jonction P-N préparée sur couche épitaxiale suivant le procédé que nous venons de décrire.

- Diode à avalanche obtenue par diffusion thermique dans le film épitaxial. La structure de la jonction est choisie de façon à permettre l'application de champs électriques intenses qui donneront lieu à une multiplication des porteurs créés par le rayonnement $[80,81]$.
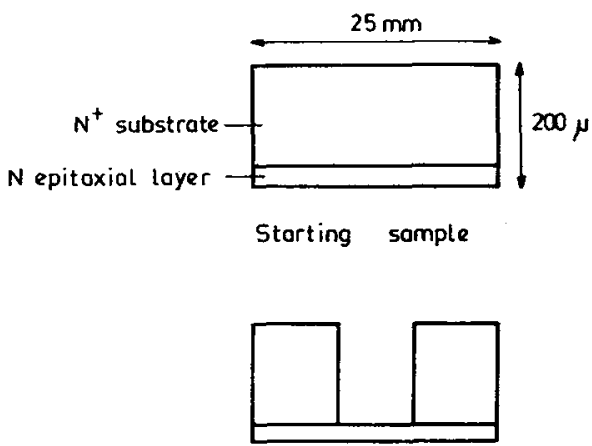

Somple ofter electrochemical etching

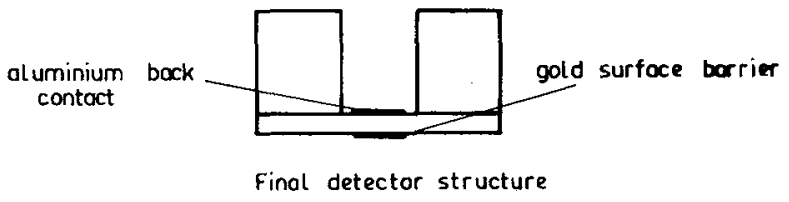

FIG. 17. - Principe de réalisation des diodes minces.

- Diode à deux couches épitaxiales $\mathbf{N}$ et $\mathbf{P}$ donnant directement une jonction enterrée [81].

- Diode à localisation, en mettant en cuvre les derniers développements technologiques de l'électronique intégrée [82].

Ce dernier type de compteur n'a pas encore eu de réalisation pratique mais il est tout à fait possible d'atteindre une résolution en position de $0,1 \mathrm{~mm}$. L'empilement de plusieurs de ces compteurs augmente encore les possibilités expérimentales.

3.2 Dimensions. - Les compteurs les plus minces réalisés à ce jour ont une épaisseur de l'ordre de $1 \mu$ et une surface limitée à $50 \mathrm{~mm}^{2}$, alors que les plus épais $(50 \mu)$ peuvent atteindre $4 \mathrm{~cm}^{2}$. L'uniformité d'épaisseur a été mesurée par plusieurs auteurs $[78,80]$ par absorption soit de photons ou de particules $\alpha$. Elle a été trouvée meilleure que $\pm 0,3 \mu$.

3.3 PROPRIÉtÉS ÉlECTRIQUES. - Le courant de fuite de ces dispositifs sera en principe identique à celui des diodes conventionnelles. Toutefois, il sera sensible à la présence éventuelle de trous dans le film, provenant d'un défaut d'empilement des atomes par suite de la présence d'une impureté physique ou chimique à la surface du substrat. De plus l'injection des porteurs devra être contrôlée par suite de l'extension de la zone de charge d'espace jusqu'au voisinage immédiat des contacts. Des courants de fuite de $0,1-1 \mu \mathrm{A}$ sont généralement obtenus pour des tensions de quelques volts et des surfaces actives de l'ordre de $50 \mathrm{~mm}^{2}$.

La capacité est évidemment inversement proportionnelle à l'épaisseur du compteur de sorte que des valeurs très importantes peuvent être atteintes pour les diodes les plus minces lorsque les surfaces sont 


\section{EPITAXIAL SILICON SURFACE BARRIER (ESSB) DESIGN}
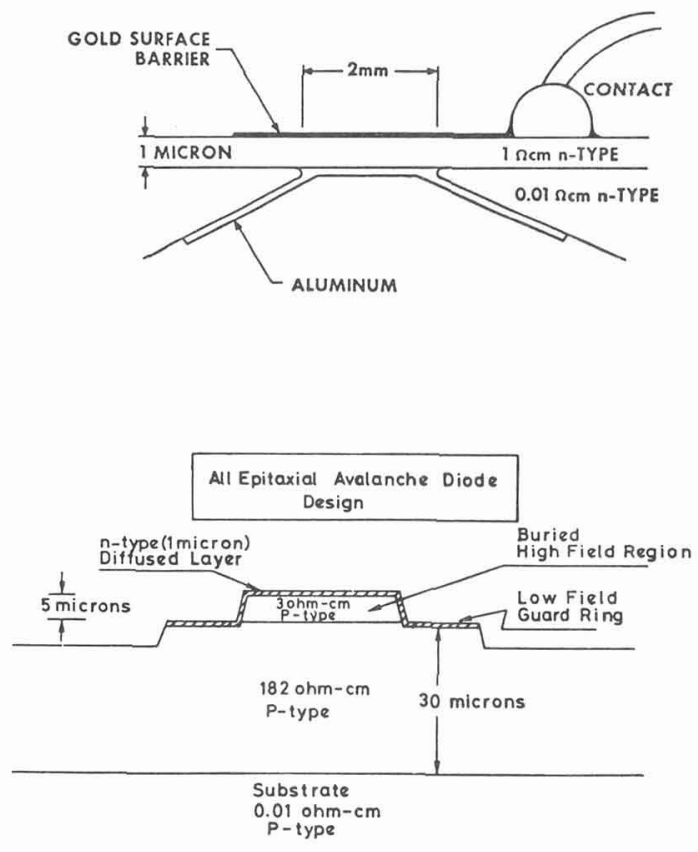

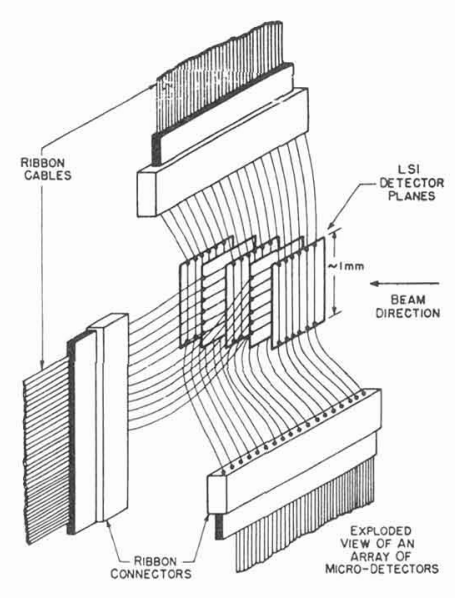

EPITAXIAL SILICON DIODE (ESD)

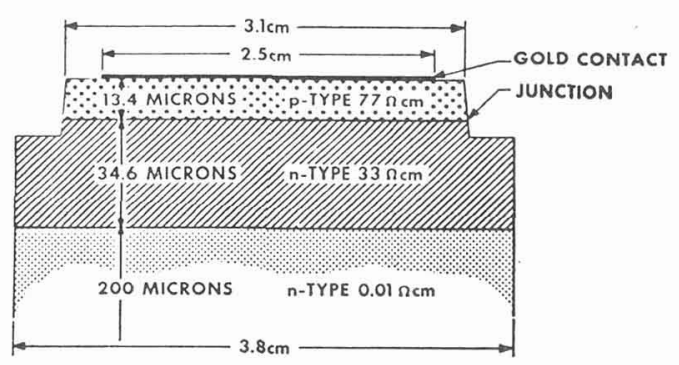

FIG. 18. - Configurations proposées de réalisation de diodes minces $\Delta E / \Delta x[80,82]$

importantes. Dans ce cas des préamplificateurs acceptant des capacités d'entrée grandes devront être employés.

3.4 Propriétés de détection. - 3.4.1 Amplitude du signal. _. Elle est évidemment fonction de la nature de la particule et de l'épaisseur du film. Sur la figure 19 nous avons reporté la réponse d'une diode de $4 \mu$ d'épaisseur à un faisceau de ${ }^{16} \mathrm{O}$ de $44,89 \mathrm{MeV}$. On notera que les phénomènes de canalisation sont considérablement réduits par une orientation convenable du détecteur par rapport au faisceau.

3.4.2 Résolution en énergie. - Le plus souvent elle est déterminée par les fluctuations de perte d'énergie et non par le bruit électronique (sauf lorsque les capacités deviennent très importantes). Plusieurs auteurs [83-85] ont calculé sa contribution. Lorsque $\Delta E / E$ est petit $(<5 \%)$ le modèle de Landau conduit à une résolution $R(\%)$ dans un film d'épaisseur $X(\mu)$ dans lequel la particule perd une énergie $\Delta E(\mathrm{MeV})$ ayant pour expression :

$$
R=1,46 \times 10^{-2} Z \frac{\sqrt{x}}{\Delta E}
$$

Pour un ion d'énergie donnée, la résolution s'améliorera donc lorsque l'épaisseur du film croît. Mais les pouvoirs d'arrêt élevés des ions lourds limitent très souvent les épaisseurs possibles.

Mentionnons également qu'à la traversée du compteur le faisceau s'élargit. L'importance de cet effet a été calculée pour des projectiles légers [86-88] et mesurée également pour des ions lourds [89].

3.4.3 Résolution en temps. - Si à l'origine l'expérimentateur exigeait essentiellement une bonne résolution en énergie des diodes à semiconducteurs, il est de plus en plus nécessaire de disposer également de bonnes informations temporelles, notamment pour les dispositifs d'identification en $Z$ et $M$.

Comme la vitesse limite de déplacement des porteurs dans le silicium atteint $10^{7} \mathrm{~cm} / \mathrm{s}$, le temps de collecte des charges $T_{c}$ dans une diode d'épaisseur $X(\mu)$ sera :

$$
T_{\mathrm{c}}(\mathrm{ps})=10 X
$$

de sorte que pour $X=10 \mu$ par exemple 100 ps suffisent à collecter les charges. Dans le cas d'ions très lourds ce temps peut être quelque peu rallongé par le temps mis par le nuage très dense de porteurs de charges de se dissocier (plasma-time) [90,91]. Un accroissement de la tension appliquée permet de le minimiser, voire de le supprimer. Reste à extraire ce signal du bruit de fond et à déclencher un discrimina- 

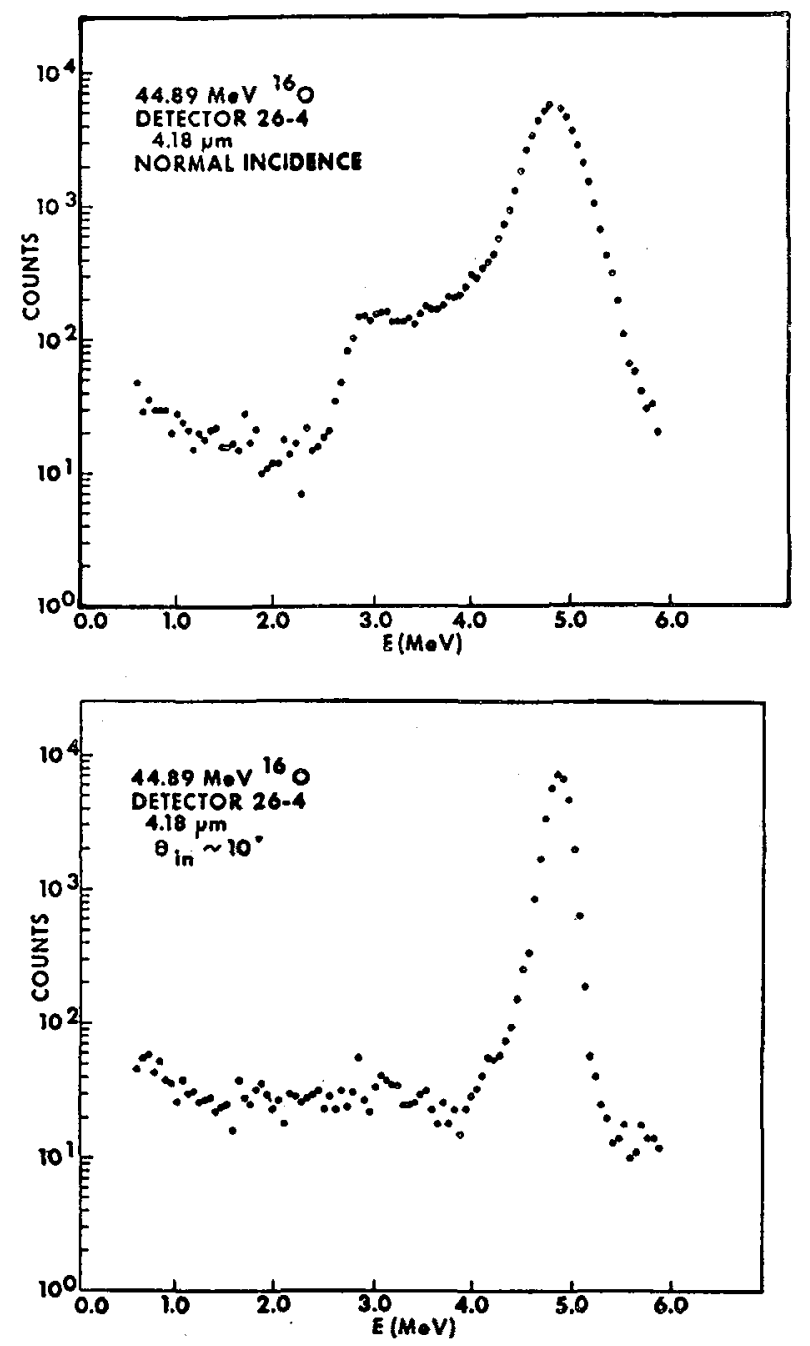

FIG. 19. - Spectre de ${ }^{16} \mathrm{O}^{+}$de $44,89 \mathrm{MeV}$ enregistré dans un détecteur $\Delta E / \Delta x$ d'épaisseur $4,18 \mu \mathrm{m}$, montrant l'effet de la canalisation d'ions.

teur au niveau le plus bas compatible avec le bruit. La résolution en temps sera donnée par:

$$
\Delta t(\mathrm{ps})=\frac{4 C}{\Delta E}\left(\frac{\tau}{g_{\mathrm{m}}}\right)^{1 / 2}
$$

où $C(\mathrm{pF})$ représente la capacité d'entrée totale du circuit, $\tau$ le temps de montée de l'amplificateur ( $\approx 1 \mathrm{~ns}$ ) et $g_{\mathrm{m}}$ la transconductance du transistor entrée FET (50 mA/V), $\Delta E$ l'énergie absorbée en $\mathrm{MeV}$.

Dans une base de temps de vol constituée d'un compteur $\Delta E$ et $E$ à semiconducteurs les résolutions en temps sont évidemment fonction de l'expérience considérée. Toutefois des largeurs à mi-hauteur de moins de $100 \mathrm{ps}$ ont été atteintes [92].

3.4.4 Radiorésistance. - Deux paramètres sont à considérer dans la dégradation des performances d'un dispositif semiconducteur sous irradiation :

- l'effet des rayonnements sur la jonction proprement dite : le contact métal semiconducteur est beaucoup plus sensible qu'une jonction implantée ou que les diodes à deux couches épitaxiales considérées ci-dessus ;

- l'effet sur le matériau de base qui se traduit par l'introduction de niveaux pièges profonds dans la bande interdite conduisant à une augmentation du courant de génération et des probabilités de capture des porteurs. Cet effet sera beaucoup moins important ici que dans les diodes conventionnelles puisque la résistivité est plus faible. Il est en effet bien connu que la radiorésistance du silicium est d'autant meilleure que sa résistivité est plus basse.

Situation de ces compteurs par rapport aux autres dispositifs minces. - Ces derniers sont essentiellement au nombre de quatre :

- les compteurs proportionnels à circulation de gaz peuvent présenter des dimensions plus importantes avec des fenêtres de l'ordre de $100 \mu \mathrm{g} / \mathrm{cm}^{2}$. Leur épaisseur équivalente d'absorption peut être facilement modifiée en jouant sur la pression du gaz. Ils ont connu un développement important au cours de ces dernières années pour l'identification de particules de $Z$ élevés ou de faible énergie. Les signaux ont des amplitudes très importantes, par contre le facteur de multiplication des charges n'est pas uniforme à travers tout le volume sensible de sorte que la résolution en énergie est nettement moins favorable ;

- les chambres d'ionisation seraient intéressantes mais le niveau de bruit et la résolution en énergie sont moins favorables (l'énergie de création d'une paire est $10 \times$ supérieure à celle exigée dans un semiconducteur);

- les scintillateurs produisent des signaux rapides mais il est délicat de les utiliser en feuilles très minces. De plus pour des ions lourds des saturations de la lumière apparaissent dégradant l'information en énergie. Les résolutions en temps dans les expériences de temps de vol sont typiquement de 100-200 ps ;

- films minces (généralement de carbone) avec détection des électrons secondaires par des multiplicateurs à microcanaux. Ce dispositif ne donne évidemment aucune information précise sur l'énergie mais il a l'avantage, vu l'épaisseur des feuilles, de n'introduire qu'une perturbation très faible dans le faisceau. Des temps de résolution de l'ordre de $100-200$ ps ont été atteints.

4. Compteurs E. - Il n'est pas possible d'aborder dans le cadre de cet exposé tous les problèmes liés à la spectrométrie d'ions lourds. Nous nous limiterons à l'ensemble des phénomènes qui sont susceptibles de modifier le comportement du compteur par rapport à la réponse qu'il donne sous bombardement de particules légères. Des méthodes d'étalonnage ont été proposées dans la littérature mettant soit en œuvre la réponse à une source de fragments de fission [93] ou au contraire ne tenant compte que de considérations théoriques [94]. Bien que ces procé- 
dés soient souvent employés par les physiciens nucléaires il convient de remarquer qu'ils sont très approximatifs, car d'autres paramètres peuvent avoir une influence non négligeable. Observons schématiquement le ralentissement d'un ion lourd dans un compteur silicium. Avant de pénétrer dans le volume sensible il doit traverser une zone morte (fenêtre d'entrée) puis il perd son énergie essentiellement par des excitations coulombiennes et des excitations et ionisations des électrons du milieu absorbant. Seuls les chocs électroniques conduisent à la création de paires électron-trou (énergie de création de paires) les autres interactions ne participant pas à la formation du signal électrique (chocs nucléaires). Les paires électron-trou sont séparées par le champ électrique avec plus ou moins d'efficacité vers les électrodes de collection, certaines peuvent disparaître (effets de plasma, recombinaison). Dans certaines conditions des multiplications de charges peuvent se produire (avalanche), de plus des défauts sont créés par des atomes de recul (dommages). Il nous semble que les points suivants doivent être pris en considération.

a) Energie de création d'une paire. _ Les modèles théoriques considèrent que l'énergie de création $\varepsilon$ est indépendante de la nature de la particule. Expérimentalement, plusieurs auteurs $[95,96]$ ont cons taté que cette énergie diminuait $(\approx 1 \%)$ pour des ions plus lourds. Toutefois, les écarts observés varient considérablement d'une expérience à l'autre de sorte que nous pensons que d'autres phénomènes ont pu être à l'origine des observations expérimentales.

b) Fenêtre d'entrée. - De nombreuses expériences ont montré que la zone morte existant à l'entrée d'un compteur silicium à barrière de surface ne se limite pas au film d'or. Elle dépend d'un grand nombre de phénomènes [97] dont la résistivité, la température, les traitements de surface... Par contre, des résultats nettement plus reproductibles sont obtenus par implantation ionique ; de plus dans ce cas la couche morte peut être nettement moins absorbante.

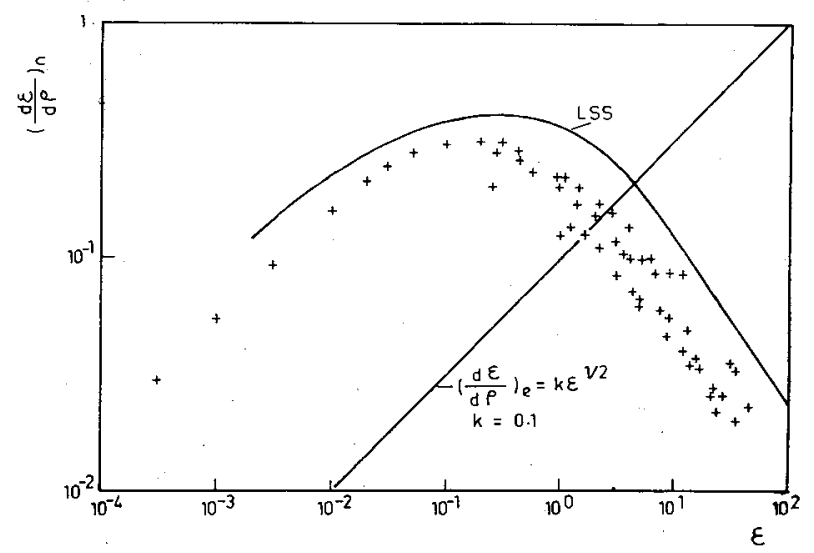

FrG. 20. - Evolution du pouvoir d'arrêt nucléaire et électronique en fonction de l'énergie en coordonnées réduites. Les points représentent les valeurs expérimentales. c) Chocs nucléaires. - Les problèmes liés à la perte d'énergie d'un ion lourd par chocs électriques et nucléaires furent d'abord considérés par Bohr [98] puis ils ont fait l'objet d'un traitement théorique approfondi par Lindhard [99-101]. Les résultats sont présentés sous forme de courbes universelles: ainsi sur la figure 20 nous avons reporté le pouvoir d'arrêt nucléaire dans les unités employées par Lindhard et sur la figure 21 l'énergie perdue en chocs nucléaires en fonction de l'énergie, les deux quantités exprimées en coordonnées réduites. Ces valeurs sont très largement employées par les physiciens nucléaires mais il convient de se rappeler qu'elles ne sont qu'approchées et que des écarts importants peuvent être observés pour les ions relativement lourds ( $>$ Si) aux énergies élevées (Figs. 22, 23) [102-103].

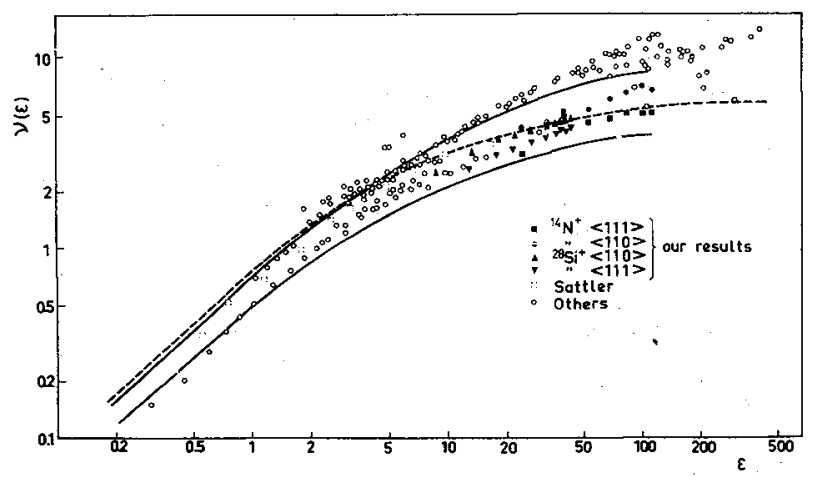

FIG. 21. - Energie perdue en collisions nucléaires en fonction de l'énergie en coordonnées réduites pour des ions ${ }^{14} \mathrm{~N}^{+}$et ${ }^{28} \mathrm{Si}^{+}$ absorbés dans le silicium.

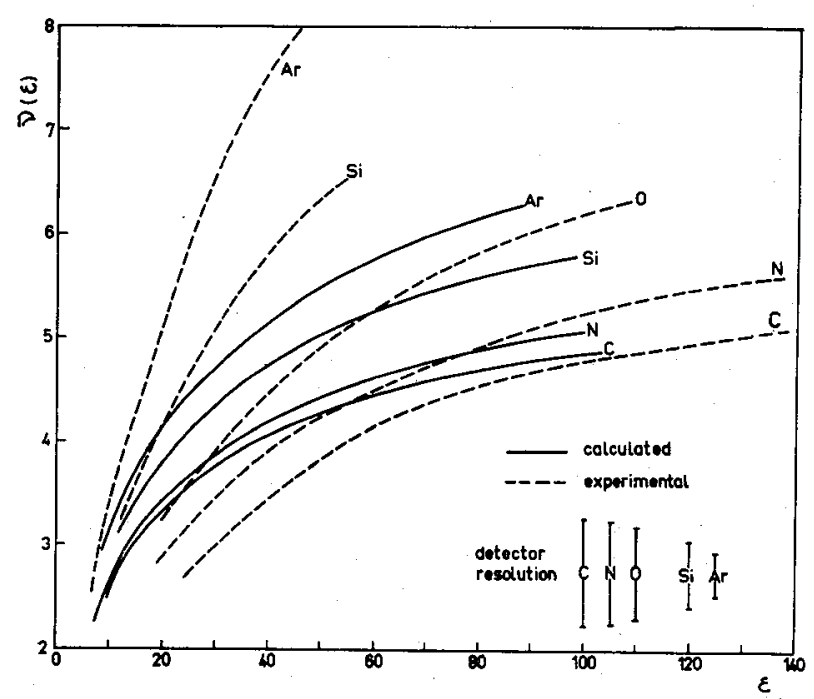

FIG. 22. - Energie perdue en collisions nucléaires par des ions $\mathrm{Ar}^{+}, \mathrm{Si}^{+}, \mathrm{O}^{+}, \mathrm{N}^{+}, \mathrm{C}^{+}$dans le silicium en fonction de l'énergie (coordonnées réduites).

d) Avalanche. - Des phénomènes de multiplication de charges apparaissent quelle que soit la nature de la particule lorsqu'une certaine densité de porteurs est dépassée et que le détecteur comporte une électrode métallique. Lorsque ces deux conditions 


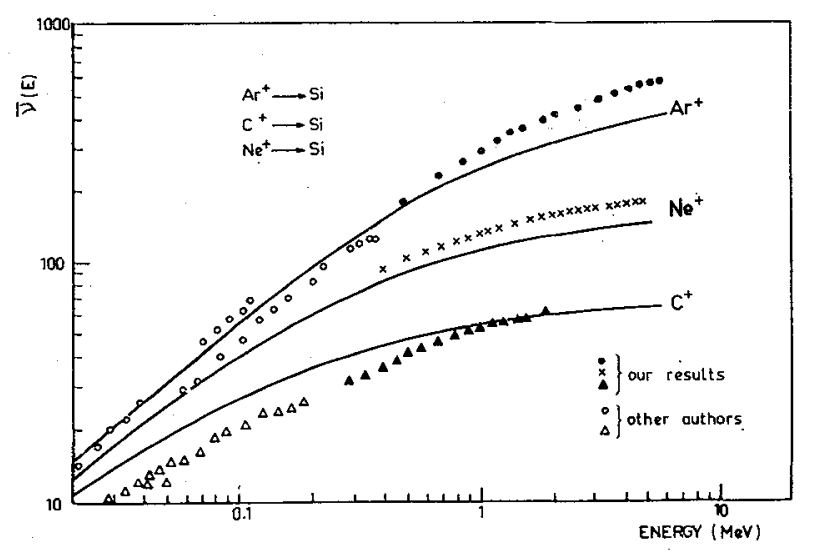

FIG. 23. - Energie perdue en collisions nucléaires dans le silicium par des ions $\mathrm{Ar}^{+}, \mathrm{Ne}^{+}, \mathrm{C}^{+}$dans un grand domaine énergétique.

sont réunies même des particules très légères peuvent donner lieu à l'avalanche (Fig. 24). Les diodes implantées ne comportant pas d'électrode métallique frontale sont exempts d'avalanche [104].

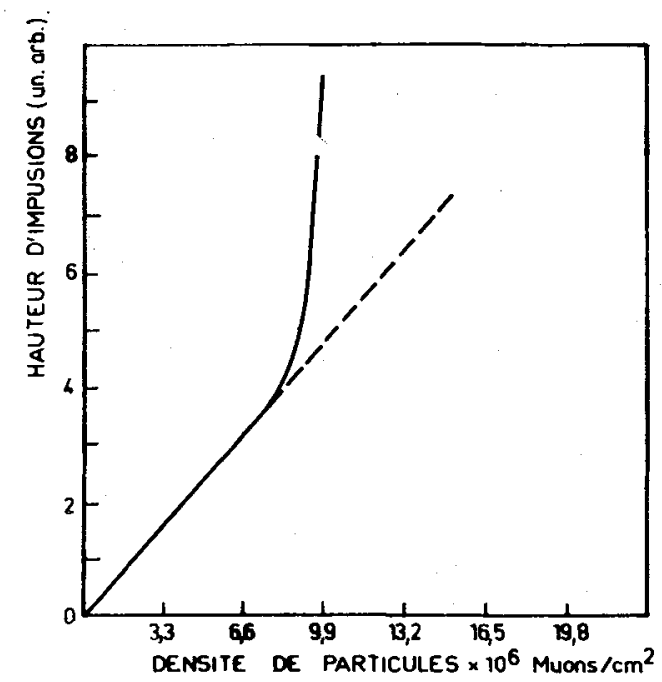

FIG. 24. - Evolution de la hauteur d'impulsion issue d'un détecteur $\mathrm{Si}$ en fonction du flux de muons [104].

e) Effet de plasma, recombinaison. - Ces problèmes ne sont pas clairs à l'heure actuelle dans la littérature car de nombreux auteurs ne considèrent pas l'ensemble des phénomènes pouvant donner lieu à une perte de porteurs et ont tendance à mettre dans cette rubrique toute la différence énergétiqué entre l'amplitude observée expérimentalement et celle calculée en ne tenant compte que des collisions nucléaires théoriques. Nos propres expériences nous incitent à croire $[102,103]$ que pour des ions allant jusqu'à $\mathrm{Ar}^{+}$de $8 \mathrm{MeV}$ cet effet peut être rendu négligeable en mettant en ouvre des compteurs de faible résistivité dans lesquels les champs électriques sont intenses. f) Dommages par radiations. - Plusieurs auteurs ont constaté que les diodes à barrière de surface se dégradent rapidement pour des doses dépassant $10^{\dagger}$ ions $/ \mathrm{cm}^{2}$, cette dégradation est encore plus rapide à basse température et se traduit tout d'abord par un allongement des temps de montée dû au piégeage de porteurs. Des expériences similaires ont été effectuées sur des compteurs implantés et leur tenue sous bombardement fut trouvée nettement meilleure $\left(10^{9}-10^{11}\right.$ ions $\left./ \mathrm{cm}^{2}\right)$ (Fig. 25) $[111]_{\dagger}$

Compte tenu de l'ensemble de ces considérations, il nous semble que les diodes implantées devraient remplacer les compteurs à barrière de surface en spectrométrie d'ions lourds.
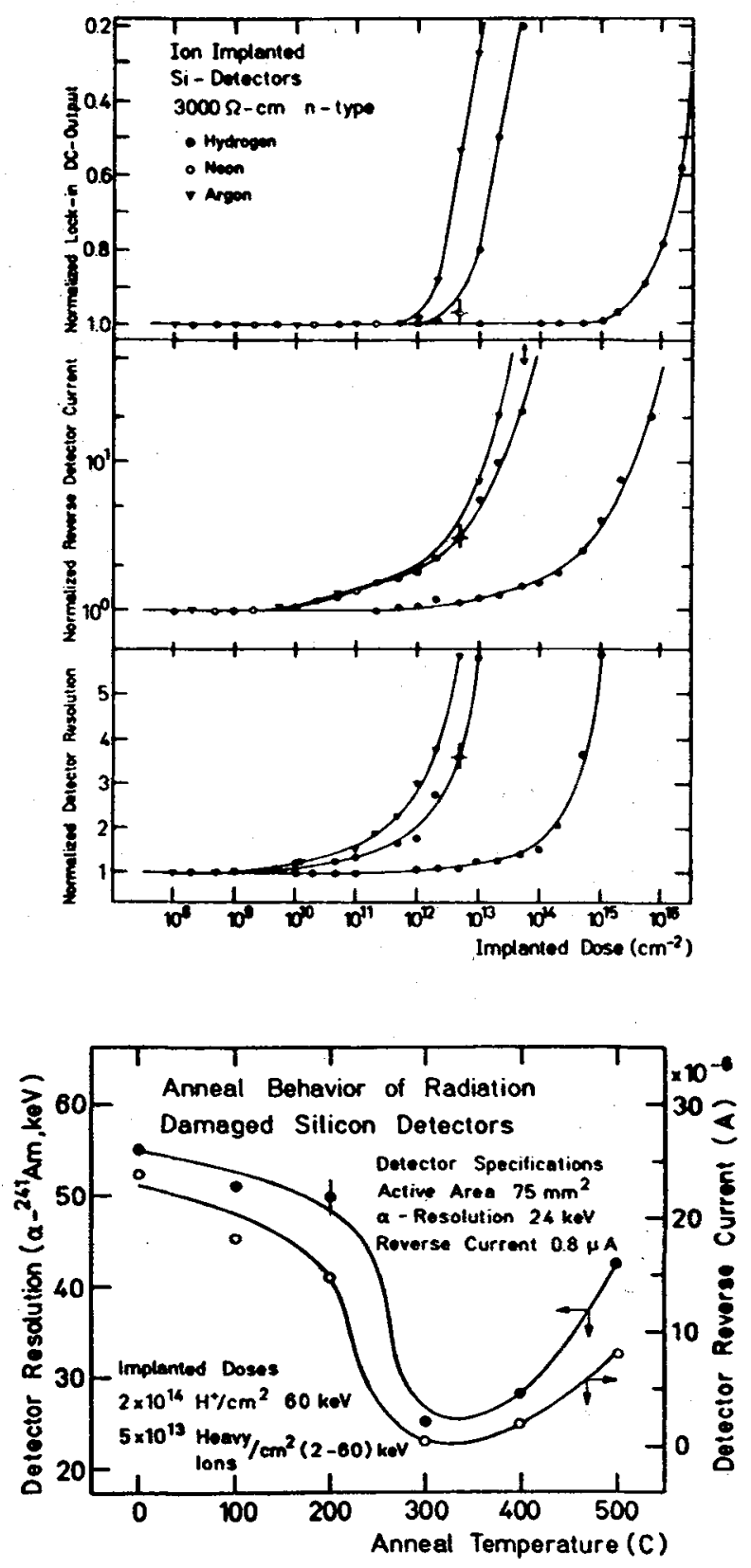

FIG. 25. - Dégradation de divers paramètres d'un détecteur silicium implanté sous divers bombardements en fonction de la dose. Guérison des défauts par recuit thermique [111]. 
5. Conclusion. - Les détecteurs à semiconducteurs après avoir pris une place importante en spectrométrie nucléaire pourront dans le futur, lorsque les compteurs $\mathrm{X}$ et $\gamma$ capables de fonctionner à température ambiante seront au point, également trouver des applications industrielles ou en médecine nucléaire. Par ailleurs, certaines expériences de physique du solide ou de physique atomique font appel à ces dispositifs.

\section{Bibliographie}

[1] Youdin, M., REICH, T., RUSINEK, H., à paraître Nucl. Instrum Methods

[2] Oppenheim, M., J. Prakt. Chem. 71 (1857) 196.

[3] Kobayashi, M., Z. Anorg. Chem. 69 (1911) 1.

[4] APPEL, J., Z. Naturforsch. 9a (1954) 265.

[5] Jenny, D. A., Bube, R. H., Phys. Rev. 96 (1954) 1190.

[6] De NoBel, D., Philips Res. Rep. 14 (1959) 361 et 430.

[7] Proceedings 1st Intern. Symp. on Cadmium Telluride Strasbourg (1971) Ed. P. Siffert, A. Cornet.

[8] Proceedings 2nd Intern. Symp. on Cadmium Telluride, Revue Phys. Appl. 12 (1977) 105 à 432.

[9] Matveev, O. A., Prokof'ev, S. V., RuD', Ya. V., Inorg. Mat. 5 (1969) 1000

[10] Triboulet, R., Thèse Paris 1972.

[11] KYLE, N. R., Réf. [7] paper IV.

[12] Medvdev, S. A., Maximovsky, S. N., Klevkov, Yu. V., CHAPNIN, P. V., Inorg. Mater. 4 (1968) 1759.

[13] Sмith, F. T., Met. Trans. 1 (1970) 617.

[14] ZANIO, K. R., Intern. Progress Report (1968) SAN-549-5.

[15] Whelan, R. C., Shaw, D., Phys. Stat. Sol. 29 (1968) 145.

[16] Schaub, B., Communication privée.

[17] Woodbury, H. H., II-VI Compounds Edité par D. G. Thomas (Benjamin, New York), 1967, p. 244.

[18] Kroger, F. A., Vink, H. J., Solid State Phys. F. Seitz ed. (Academic Press, New York) 1956, p. 310.

[19] Mandel, G., Phys. Rev. 134 (1964) 1073.

[20] Brebrick, R. F., J. Phys. Chem. Sol. 4 (1958) 190.

[21] Bell, R. O., Wald, F. V., Canali, C., Nava, F., Otta. VIANI, G., IEEE Trans. Nucl. Sci. NS 21 (1974) 331

[22] Kroger, F. A., J. Phys. Chem. Solids 26 (1965) 1717.

[23] Stuck, R., CORNet, A., SCHARAGER, C., Siffert, P., J. Phys. Chem. Solids 37 (1976) 989.

[24] HeCHT, K., Z. Phys. 77 (1932) 235.

[25] Dabrowski, A. J., Chwaszczewska, J., Iwanczyk, J., TRIboulet, R., Marfaing, Y., Revue Phys. Appl. 12 (1977) 297.

[26] Cornet, A., Hage-Ali, M., Grob, J. J., StucK, R., SifFERT, P., IEEE Trans. Nucl. Sci. NS 19 (1972) 358.

[27] Agrinskaya, N. V., Arkad'eva, E. N., Matveev, O. A., Ryvkin, S. M., Sladkova, V. A., Starinin, K. V., Réf. [7] paper XVI.

[28] Malm, H. L., Canali, C., Mayer, J. W., Nicolet, M. A., Zanio, K. R., Akutagawa, W., Appl. Phys. Lett. 26 (1975) 344, voir aussi Réf. [8] p. 303.

[29] Ukrainets, V. E., Kurilo, I. V., Palivoda, I. P., Sov. Phys. Semicond. 10 (1976) 838.

[30] ARKadeva, E. N., Maslova, L. V., Matveev, O. A., Rud', Yu. V., Ryukin, S. M., Sov. Phys. Semicond. 1 (1967) 669.

[31] MAYER, J. W., Semiconductor Detectors Eds G. Bertolini, A. Coche (North Holland, Amsterdam) 1968.

[32] Kasherininov, P. G., Matveev, O. A., Maslova, L. M., Sov. Phys. Semicond. 4 (1966) 451.

[33] CORNET, A., Thèse Strasbourg (1976).

[34] Rabin, B., Tabatabal, H. Y., Siffert, P., à paraître.

[35] MaLm, H. L., MARTINI, M., IEEE Trans. Nucl. Sci. NS 21 (1974) 322.

[36] Fabre, E., Ngo-Tich-Phuoc, Martin, G. M., ORTEGa, F., IEEE Trans. Nucl. Sci. NS 23 (1976) 182.

[37] SIFFERT, P., RABIN, B., TABATABAi, H. Y., Stuck, R., à paraître Nucl. Instrum. Methods.

[38] ZANIO, K. R., Réf. [8] p. 343.

[39] SIFFERT, P., à paraître Nucl. Instrum. Methods.

[40] Cornet, A., Siffert, P., Coche, A., Truboulet, R., Appl. Phys. Lett. 17 (1970) 432.
[41] Dabrowski, A. J., Iwancsyk, J., Triboulet, R., Nucl. Instrum. Methods 118 (1974) 531.

[42] Alberigi-Quaranta, A., Canali, C., Otaviani, O., ZANIO, K. R., Nuovo Cimento Lett. 4 (1970) 908.

[43] Stuck, R., Ponpon, J. P., Berger, R., Siffert, P., Radiat. Eff. 20 (1973) 75.

[44] JAGER, H., THIEL, R., Réf. [8] p. 293.

[45] Sherman, J., Spectrochim. Acta 7 (1955) 283.

[46] Akutagawa, W., Zanio, K. R., J. Appl. Phys. 40 (1969) 3838 .

[47] Zanio, K. R., Akutagawa, W., Kikushi, R., J. Appl. Phys. 39 (1968) 2296.

[48] MaYer, J. W., Chap. 5 Semiconductor Detectors Eds G. Bertolini, A. Coche (North Holland) 1968.

[49] Kiein, C. A., Proc. Intern. Conf. Semicond. Kyoto (1966) 307, J. Appl. Phys. 39 (1968) 2029.

[50] KURZ, R., à paraître Nucl. Instrum. Methods.

[51] ZANIO, K. R., Rapport AT (94-3) 549 (1975).

[52] Day, R. B., Dearnaley, G., Palms, J., IEEE Trans. Nucl. Sci. NS 17 (1970) 176.

[53] Trammel, R., Walter, F. J., Nucl. Instrum. Methods 76 (1969) 367.

[54] ARMANTROUT, G. A., UCRL Rapport 50485 (1970).

[55] Hofstadter, R., Nucleonics 4 (1949) 3.

[56] Siffert, P., Hage-Ali, M., Stuck, R., Cornet, A., Réf. [8] p. 335.

[57] Siffert, P., Berger, R:, Stuck, A., Bell, R. O., Wald, F., IEEE Trans. Nucl. Sci. NS 23 (1976) 159.

[58] KikUCHI, C., Radiat. Eff. 8 (1971) 249.

[59] NAKano, G. H., Simpson, D. A., IMHOF, L., IEEE Trans. Nucl. Sci. NS 23 (1976) 468.

[60] CORnET, A., Siffert, P., non publié.

[61] Nikitine, S., Sieskind, M., C.R. Hebd. Séan. Acad. Sci. 240 (1955) 1324

Nikitine, S., Prog. Semicond. 6 (1962) 269.

[62] Sieskind, M., Thèse Strasbourg (1960) Rev. Opt. 39 (1960) 239.

[63] Gross, E. F., Kaplyansku, A. A., Zh. Tekh. Fiz. 25 (1955) 2061.

[64] Bube, R. H., Phys. Rev. 106 (1957) 703

[65] Novikov, B. V., Pimonenko, M. M., Sov. Phys. Sem. 4 (1971) $1285 ; 6$ (1972) 671 .

[66] Chepur, D. V., Petrusevick, V. A., Z. Tekh. Fiz. 25 (1955) 1523 ; Chem. Abstr. 50 (1956) 50.

[67] WILlig, W. R., Réf. [8] paper XXVII, Nucl. Instrum. Methods 96 (1971) 615; 101 (1972) 23, Rapport Interne Siemens 2 (3) (1973) 157.

[68] Workshop on Mercuric Iodide Strasbourg (1975) Eds. P. Siffert, J. P. Ponpon, I. Zchokke, C. Schwab.

[69] WhITED, R. C., VAN DEN BERG, L., IEEE Trans. Nucl. Sci. NS 241 (1977) 165.

[70] Ponpon, J. P., ST́uck, R., Siffert, P., Schwab, C., Nucl. Instrum. Methods 119 (1974) 197; IEEE Trans. Nucl. Sci. NS 22 (1975) 182.

[71] Llacer, J. L., WatT, M. K., Schieber, M., Carlson, M. SCHNEPPLE, W., IEEE Trans. Nucl. Sci. NS 21 (1974) 305.

[72] Randtke, P. T., Ortale, C., Whited, C., Van den Berg, L., sous presse.

[73] Randtke, P. F., Ortale, C., IEEE Trans. Nucl. Sci. NS 24 (1977) 129.

[74] RandtKe, P. T., ORtale, C., Whited, R. C., VAN DEN BERG, L., Proc. ERDA Symposium on X-ray and $\gamma$-ray sources and Applications (1976) Ann Arbor, USA. 
[75] SeibT, W., Slapa, M., HUTH, G., Nucl. Instrum. Methods 135 (1976) 573.

[76] Schieber, M., Workshop on Mercuric Iodide, Jerusalem (1977), à paraître Nucl. Instrum. Methods.

[77] SwIERKowski, S. P., IEEE Trans. Nucl. Sci. NS 23 (1976) 131.

[78] PONPON, J. P., SIFFERT, P., VAZEILle, F., Nucl. Instrum. Methods 112 (1973) 465.

[79] MEEK, R. L., GrBSON, W. M., BRAUN, R. H., Nucl. Instrum. Methods 94 (1971) 435.

[80] GrUHN, C. R., IEEE Trans. Nucl. Sci. NS 24 (1977) 93.

[81] Maggiore, C. J., Goldstone, P. D., Gruhn, C. R., IEEE Trans. Nucl. Sci. NS 24 (1977) 104.

[82] KANOFSKY, A., Nucl. Instrum. Methods 140 (1977) 429.

[83] LANDAU, L., $J$. Phys. USSR 8 (1944) 201.

[84] SYMON, K. R., Thèse Harvard (1948).

[85] Vavilov, P. N., Zh. Exp. i. Theo. Fiz. 32 (1957) 920, et JETP (1957) 742.

[86] Meyer, L., Phys. Stat. Sol. $44 b$ (1971) 253.

[87] Williams, E. J., Proc. Ray. Soc. 169 (1939) 531.

[88] Molmere, G., Z. Naturforsch. 2a (1947) 133 ; 3a (1948) 78.

[89] Spahn, G., Groenland, K. O., Nucl. Instrum. Methods 123 (1975) 425.

[90] Alberighi-Quaranta, A., TARoni, A., Zanarini, G., Nucl. Instrum. Methods 72 (1969) 72.

[91] SeibT, W., Sundstrom, K. E., Tove, P. A., Nucl. Instrum. Methods 113 (1973) 317.

[92] ZeIDMAN, B., HENNING, W., Kouar, D. G., Nucl. Instrum. Methods 118 (1974) 361.

[93] SchmitT, H. W., Kiker, W. I., Williams, C. W., Phys. Rev. 137 (1965) B 837.
[94] KaUfmaN, S. B., Steinberg, E. P., Nucl. Instrum. Methods 115 (1974) 47.

[95] KEMPER, Fox, Nucl. Instrum. Methods 105 (1972) 333.

[96] LANGLEY, Nucl. Instrum. Methods 113 (1973) 109.

[97] Mitchel, AGAmi, Fox, Radiat. Eff. 28 (1976) 133.

[98] Bohr, N., Mat. Fys. Medd. Dan. Vid. Selsk. 18 n $^{\circ} 8$ (1954).

[99] LindhaRd, J., NiElsen, V., ScharfF, M., Mat. Fys. Medd. Dan. Vid. Selsk 36 (1968) no 10.

[100] Lindhard, J., SCharfF, M., SCHiøTt, H. E., Mat. Fys. Medd. Dan. Vid. Selsk 33 (1963) no 14.

[101] Lindhard, J., Nielsen, V., SCharfF, M., THOMSEN, P. U., Mat. Fys. Medd. Dan. Vid. Selsk 33 (1963) no 10.

[102] Grob, J. J., Grob, A., Pape, A., Siffert, P., Phys. Rev. B 11 (1975) 3273

[103] GroB, J. J., GRoB, A., SIFFERT, P., Nucl. Instrum. Methods 132 (1976) 273.

[104] Heijne, H. M., Muller, J. C., Siffert, P., à paraître IEEE.

[105] Canali, C., Nava, F., Ottaviani, G., Zanio, K. R., Solid State Commun. 13 (1973) 1255 ; Phys. Stat. Sol. a 28 (1975) 581 .

[106] Triboulet, R., Réf. [8] p. 297.

[107] TaguChi, T., ShIRafuII, J., INUiShi, Y., Réf. [8] p. 117.

[108] Martin, G., BACK, P., TrancharT, J. C., TABre, E., IEEE Trans. Nucl. Sci. NS 22 (1975) 226 ; NS 23 (1976) 154.

[109] STuck, R., Thèse Strasbourg (1976).

[110] Alekseenko, M. V., Sov. Phys. Sem. 8 (1974) 351.

[111] KALBITZER, S., communication privée. 